\title{
Incentive and dopamine sensitization produced by intermittent but not long access cocaine self-administration
}

\author{
Alex B. Kawa ${ }^{1}$ (D) | Alec C. Valenta ${ }^{2} \quad$ Robert T. Kennedy ${ }^{2} \quad$ Terry E. Robinson ${ }^{1}$
}

${ }^{1}$ Department of Psychology (Biopsychology Program), University of Michigan, Ann Arbor, Michigan

${ }^{2}$ Department of Chemistry, University of Michigan, Ann Arbor, Michigan

\section{Correspondence}

Terry E. Robinson, Department of Psychology, University of Michigan, 530 Church Street, East Hall, Ann Arbor, MI 48109.

Email: ter@umich.edu

Funding information

National Institute of Biomedical Imaging and Bioengineering, Grant/Award Number: EB003320; National Institute on Drug Abuse, Grant/Award Number: DA007281, DA031656 and DA044204

\begin{abstract}
The temporal pattern of drug use (pharmacokinetics) has a profound effect on the ability of self-administered cocaine to produce addiction-like behavior in rodents, and to change the brain. To further address this issue, we compared the effects of long access $(\operatorname{LgA})$ cocaine self-administration, which is widely used to model the transition to addiction, with intermittent access (IntA), which is thought to better reflect the pattern of drug use in humans, on the ability of a single, self-administered injection of cocaine to increase dopamine (DA) overflow in the core of the nucleus accumbens (using in vivo microdialysis), and to produce addiction-like behavior. IntA experience was more effective than $\operatorname{LgA}$ in producing addiction-like behavior-a drug experience-dependent increase in motivation for cocaine assessed using behavioral economic procedures, and cue-induced reinstatement—despite much less total drug consumption. There were no group differences in basal levels of DA in dialysate [DA], but a single self-administered IV injection of cocaine increased [DA] in the core of the nucleus accumbens to a greater extent in rats with prior IntA experience than those with $\mathrm{LgA}$ or limited access experience, and the latter two groups did not differ. Furthermore, high motivation for cocaine was associated with a high [DA] response. Thus, IntA, but not $\operatorname{LgA}$, produced both incentive and DA sensitization. This is consistent with the notion that a hyper-responsive dopaminergic system may contribute to the transition from casual patterns of drug use to the problematic patterns that define addiction.
\end{abstract}

\section{K E Y W O R D S}

addiction, cocaine, dopamine, intermittent access, sensitization

\section{INTRODUCTION}

Drug self-administration procedures have proved invaluable for isolating how drug use can change the brain in ways that promote the development of addiction-like behavior (Ahmed, 2018; Weeks, 1962). However, mere drug-taking does not necessarily produce the loss of control and aberrant motivation that defines addiction. For this reason, there has been considerable interest in the development of self-administration procedures and conditions (here we focus on cocaine) that most reliably produce addiction-like behavior (Ahmed, 2012; Ahmed \& Koob, 1998; Deroche-Gamonet, Belin, \&

Abbreviations: DA, dopamine; IC, infusion criteria; IntA, intermittent access; LgA, long access; LimA, limited access.

Edited by Michel Barrot. Reviewed by Steve Mahler, Pascal Romieu and Anonymous.

All peer review communications can be found with the online version of the article. 
Piazza, 2004; Vanderschuren \& Everitt, 2004; Zimmer, Oleson, \& Roberts, 2012). To this end, Ahmed and Koob (1998) first introduced what has become known as the long access ( $\operatorname{LgA})$ self-administration procedure, which is arguably the most widely used rodent model of addiction. During $\mathrm{LgA}$, rats are typically allowed to self-administer cocaine on a fixed ratio (FR) schedule for $6+$ hr/daily session; and relative to rats allowed short, or more limited access $(\operatorname{LimA})$ to cocaine (1-2 hr/session), $\mathrm{LgA}$ experience is reported to result in the emergence of a number of addiction-like behaviors, including escalation of intake, increased motivation for drug, and continued drug-seeking in the face of an adverse consequence (for reviews see, Ahmed, 2012; Edwards \& Koob, 2013; Kawa, Allain, Robinson, \& Samaha, 2019).

Studies on the neurobiological consequences of LgA cocaine experience suggest that $\operatorname{LgA}$ decreases dopamine (DA) neurotransmission in the nucleus accumbens core (relative to rats with more limited experience), at least when tested soon after the discontinuation of self-administration. For example, $\mathrm{LgA}$ experience decreases cocaine-induced inhibition of DA uptake, electrically evoked DA release when measured ex vivo, and cocaine-induced DA overflow in vivo measured with microdialysis (Calipari, Ferris, \& Jones, 2014; Calipari, Ferris, Zimmer, Roberts, \& Jones, 2013; Ferris, Mateo, Roberts, \& Jones, 2011). In addition, LgA cocaine self-administration experience has been associated with a progressive decrease in nucleus accumbens DA release accompanying operant responding measured using voltammetry in vivo (Willuhn, Burgeno, Groblewski, \& Phillips, 2014). Although there are exceptions (e.g., Ahmed, Lin, Koob, \& Parsons, 2003), these findings have been interpreted as support for the idea that cocaine addiction is due, in part, to a cocaine-induced hypo-dopaminergic state, and continued drug-seeking is motivated by a desire to overcome this DA deficiency (e.g., Caprioli, Calu, \& Shaham, 2014; Koob \& Volkow, 2016; U.S. Department of Health and Human Services, 2016; Volkow, Koob, \& McLellan, 2016).

A fundamental assumption of the $\operatorname{LgA}$ model is that the amount of drug exposure is the critical factor in the emergence of addiction-like behavior. As stated by Ahmed (2012), "addiction-causing neuropathological processes could be set in motion only when rats can expose themselves sufficiently to cocaine to cross the 'threshold of addiction' ... below this critical level of cocaine exposure, there would be no druginduced neuropathological changes, and drug use would remain under control, at least in the majority of drug-exposed individuals" (p. 110). However, studies using a more recently introduced model of the transition to cocaine addiction have challenged this assumption (Allain, Minogianis, Roberts, \& Samaha, 2015; Kawa et al., 2019; Zimmer, Dobrin, \& Roberts, 2011; Zimmer et al., 2012).

During a LgA session, rats first "load up" and then maintain relatively high brain concentrations of cocaine for the duration of the $6+\mathrm{hr}$ session. However, Zimmer et al. (2012) noted that this may not reflect the pattern of cocaine use in humans, which is much more intermittent both between and within bouts of use. Thus, Zimmer et al. (2012) developed what they called the intermittent access (IntA) cocaine self-administration procedure to better model the repeated spikes in brain cocaine concentrations thought to reflect the pattern of use in humans within a bout of use, especially during the transition to addiction. To this end, rats were allowed $5 \mathrm{~min}$ of unlimited access to cocaine followed by a $25 \mathrm{~min}$ period when drug was not available, and this repeated throughout the session, resulting in successive spikes in brain cocaine concentrations. The important finding was that IntA experience produced greater motivation for cocaine than $\mathrm{LgA}$, despite much less total drug consumption. There are now a number of reports that IntA not only increases motivation for cocaine to a greater extent than $\operatorname{LgA}$, but is more effective in producing a number of other addiction-like behaviors (Allain, Bouayad-Gervais, \& Samaha, 2018; Allain, Roberts, Lévesque, \& Samaha, 2017; Allain \& Samaha, 2018; James et al., 2018; Kawa, Bentzley, \& Robinson, 2016; Kawa \& Robinson, 2019).

Furthermore, unlike LgA, which blunts DA neurotransmission, several studies have suggested that IntA does exactly the opposite; it sensitizes DA neurotransmission. For example, IntA experience is reported to increase the ability of cocaine to inhibit DA uptake and to increase electrically evoked DA release in brain slices (Calipari et al., 2013). Of course, this raises questions concerning the role of a hypo- versus hyper-dopaminergic state in addiction, especially given that IntA is more effective than $\mathrm{LgA}$ in producing addiction-like behavior. However, to date, the only studies on the effects of IntA on DA neurotransmission have been in brain slices, ex vivo. Therefore, given the theoretical implications, it is important to determine whether IntA cocaine experience produces similar effects in freely moving rats, in vivo. That was the purpose of the present experiment, in which we used in vivo microdialysis in freely moving rats to quantify changes in [DA] (and other analytes) in response to a single self-administered injection of cocaine, following $\operatorname{LimA}, \operatorname{LgA}$, or IntA cocaine self-administration experience.

\section{MATERIALS AND METHODS}

A total of 50 male Sprague-Dawley rats (Envigo, Haslett, MI) weighing $250-275 \mathrm{~g}$ upon arrival were housed individually in a climate-controlled colony room, on a reverse 12-hr light/12hr dark cycle (lights on at 20:00). Rats were housed individually to prevent one rat from irritating the surgical site and catheter port of another rat. Individually housing rats is considered a stressor and may have affected their motivation for cocaine, although the effect of social isolation in male rats on cocaine self-administration largely depends on experimental 
conditions (Boyle, Gill, Smith, \& Amit, 1991; Bozarth, Murray, \& Wise, 1989; Phillips et al., 1994; Schenk, Lacelle, Gorman, \& Amit, 1987; for review see, Lu, Shepard, Scott Hall, \& Shaham, 2003) and in this study it is unlikely to have differentially affected the groups. All testing was conducted during the 12-hr lights off period. After arrival, rats were given 1 week to acclimate to the colony room before surgery. Water and food were available ad libitum until 2 days before the first day of self-administration, at which point the rats were mildly food restricted to maintain a stable body weight for the remainder of the experiment (20-24 g/day). There is evidence that maintaining adult, male rats at a stable body weight is more healthy than ad libitum feeding (Rowland, 2007). Although food restriction affects cocaine self-administration (Carroll, France, \& Meisch, 1981; De Vry, Donselaar, \& Van Ree, 1989), it is unlikely to have differentially affected the groups in this study. Furthermore, the procedure used here did not reduce body weight, but maintained a stable body weight. All procedures were approved by the University of Michigan Committee on the Use and Care of Animals (UCUCA).

\section{1 | Apparatus}

Behavioral testing was conducted in standard $(22 \times 18 \times 13 \mathrm{~cm})$ test chambers (Med Associates, St Albans, VT) located inside sound-attenuating cabinets. A ventilating fan masked background noise. Within the test chambers, two nose poke ports were located $3 \mathrm{~cm}$ above the floor on the left and right side of the front wall, and one port was designated active and the other inactive (side counter-balanced across chambers). A red house light was located at the top, center of the back wall opposite the nose ports. During selfadministration, intravenous cocaine infusions were delivered by a pump mounted outside the sound attenuating cabinet, through a tube connected to the rat's catheter back port. The infusion tube was suspended into the chamber via a swivel mechanism, allowing the rat free movement. All measures were recorded using Med Associates software.

Microdialysis test sessions were conducted in separate but identical chambers to those described above. The only difference was that the swivel system in these boxes allowed for the microdialysis inlet tubing, outlet tubing, and the drug delivery tubing to be connected simultaneously.

\subsection{Intravenous catheter surgery}

Rats underwent intravenous catheter surgery as described previously (Crombag, Badiani, Maren, \& Robinson, 2000). Briefly, rats were anesthetized using ketamine hydrochloride (90 mg/kg i.p.) and xylazine (10 mg/kg i.p.) and a catheter was inserted into the right jugular vein and tubing was run subcutaneously to a port located on the rat's back. Rats were administered the analgesic carprofen $(5 \mathrm{mg} / \mathrm{kg}$ s.c.) once immediately prior to surgery and once each day on the first and second days post-surgery. Following surgery, catheters were flushed daily with $0.2 \mathrm{ml}$ sterile saline containing $5 \mathrm{mg} / \mathrm{ml}$ gentamicin sulfate (Vedco, MO). Catheter patency was tested periodically with intravenous injection of $0.1 \mathrm{ml}$ methohexital sodium $(10 \mathrm{mg} / \mathrm{ml}$ in sterile water; JHP Pharmaceuticals). If a rat did not become ataxic within $10 \mathrm{~s}$ of the injection, the catheter was considered not patent and the rat was removed from the study. No rat was tested with methohexital more than three times and the tests for patency were always done at least $16 \mathrm{hr}$ before the next self-administration session.

\section{3 | Self-administration: acquisition}

Rats were given $\sim 7$ days to recover from the catheter surgery, and then self-administration training commenced (Figure 1a). The rats were placed into the chamber with the house light illuminated. After $2 \mathrm{~min}$, the house light was extinguished and this signaled the beginning of each session. At that time, a nose poke into the active port, detected by an infrared photo beam, resulted in an intravenous infusion of cocaine hydrochloride (NIDA) dissolved in $0.9 \%$ sterile saline $(0.4 \mathrm{mg} / \mathrm{kg}$ per infusion, weight of the salt, in $50 \mu$ delivered over $2.6 \mathrm{~s}$ ) on a fixed ratio-1 (FR-1) schedule. Each infusion was paired with the illumination of a cue light in the active nose port for $20 \mathrm{~s}$. Nose pokes during this $20 \mathrm{~s}$ period were recorded but had no consequences. An inactive nose port was also present at all times and pokes there were recorded but had no consequences. To ensure that during initial training all rats received the same amount of cocaine and cue exposure an infusion criteria (IC) procedure was used, as described previously (Saunders \& Robinson, 2010). During these acquisition sessions, session length was determined by how long it took each rat to reach the predetermined number of infusions, not by an explicit time limit. Each rat had two sessions at IC10 (10 infusions), three sessions at IC20, and four sessions at IC40. If a rat did not reach the IC within $6 \mathrm{hr}$, the session was terminated. If a rat failed to self-administer all of the infusions available in $6 \mathrm{hr}$ on two consecutive days, that rat was excluded from further testing. In addition, if a rat failed to make twice as many active nose pokes as inactive nose pokes on each of the last three acquisition sessions that rat was given up to three additional sessions, and if the rat did not reach criteria in those three sessions it was excluded from further testing. A total of five rats were excluded during acquisition training because they failed to reach the IC or failed to discriminate between the active nose port and the inactive nose port.

\subsection{Self-administration: within-session threshold procedure}

The day after the final acquisition session, all rats were trained on a within-session threshold procedure and demand curves 
were generated, as described previously (Bentzley, Fender, \& Aston-Jones, 2013; Kawa et al., 2016). Briefly, each session (one per day) was 110 min in length, FR-1 throughout, and every $10 \mathrm{~min}$ the dose of drug was decreased on a quarter logarithmic scale $(1.28,0.72,0.40,0.23,0.13,0.072,0.040$, $0.023,0.013,0.007,0.004 \mathrm{mg} / \mathrm{kg}$ per infusion). During the threshold procedure, the nose port cue light was illuminated for the duration of each infusion. Rats could take another infusion immediately after the preceding one (i.e., the only time out period was during the duration of the infusion).

The threshold procedure yields a number of behavioral economic metrics. $Q_{0}$ is a theoretical measure of consumption when no effort is required; that is, an inherent extrapolation of the individual's consumption at very low prices (Bentzley et al., 2013; Hursh \& Silberberg, 2008; Oleson, Richardson, $\&$ Roberts, 2011). $P_{\mathrm{Max}}$ is defined as the price that elicits maximum responding; that is, the maximum price (in effort) an individual is willing to pay to maintain $Q_{0}$ (Bentzley et al., 2013; Hursh, 1991). Consumption remains relatively stable at prices lower than $P_{\text {Max }}$ but falls rapidly at prices higher than $P_{\text {Max }}$. Finally, $\alpha$ is a measure of demand elasticity and is equivalent to the slope of the demand curve (Bentzley et al., 2013; Hursh \& Silberberg, 2008). Demand elasticity describes how susceptible the consumption of a commodity is to changes in cost. Motivation is inversely proportional to $\alpha$, meaning a larger $\alpha$ value corresponds to lower motivation. Both $P_{\mathrm{Max}}$ and $\alpha$ measure the extent to which an animal is willing to work to defend their preferred level of cocaine intake $\left(Q_{0}\right)$ that is achieved at a very low cost (in effort). Each rat was tested daily for at least four sessions and until it produced three consecutive sessions with less than $25 \%$ variation in $\alpha$. For baseline data analysis $\alpha, P_{\mathrm{Max}}$, and $Q_{0}$ values (see below) were averaged over these last three sessions for each rat. A total of three rats were excluded for failure to stabilize or loss of catheter patency.

Following the baseline threshold procedure, all rats were assigned to one of three groups: LimA $(n=10)$, prolonged IntA $(n=20)$, or prolonged $\operatorname{LgA}(n=12)$. Groups were assigned following the baseline threshold test, so that there were no group differences in baseline measures of $\alpha, P_{\text {Max }}$, or $Q_{0}$. Following the baseline threshold procedure, rats in the LimA group underwent surgery to implant a microdialysis guide cannula (described below). After 3 days of recovery, each rat in the LimA group was habituated to the microdialysis chamber in which they would be tested for $\sim 1 \mathrm{hr}$. Then, these rats were given three more IC40 self-administration sessions and at least one of these sessions was conducted in the microdialysis chamber in which they would be tested, so each rat had at least one habituation session and one selfadministration session in the microdialysis chamber. Thus,

\section{Self-administration experience}

(a)

Prolonged access animals- IntA or LgA
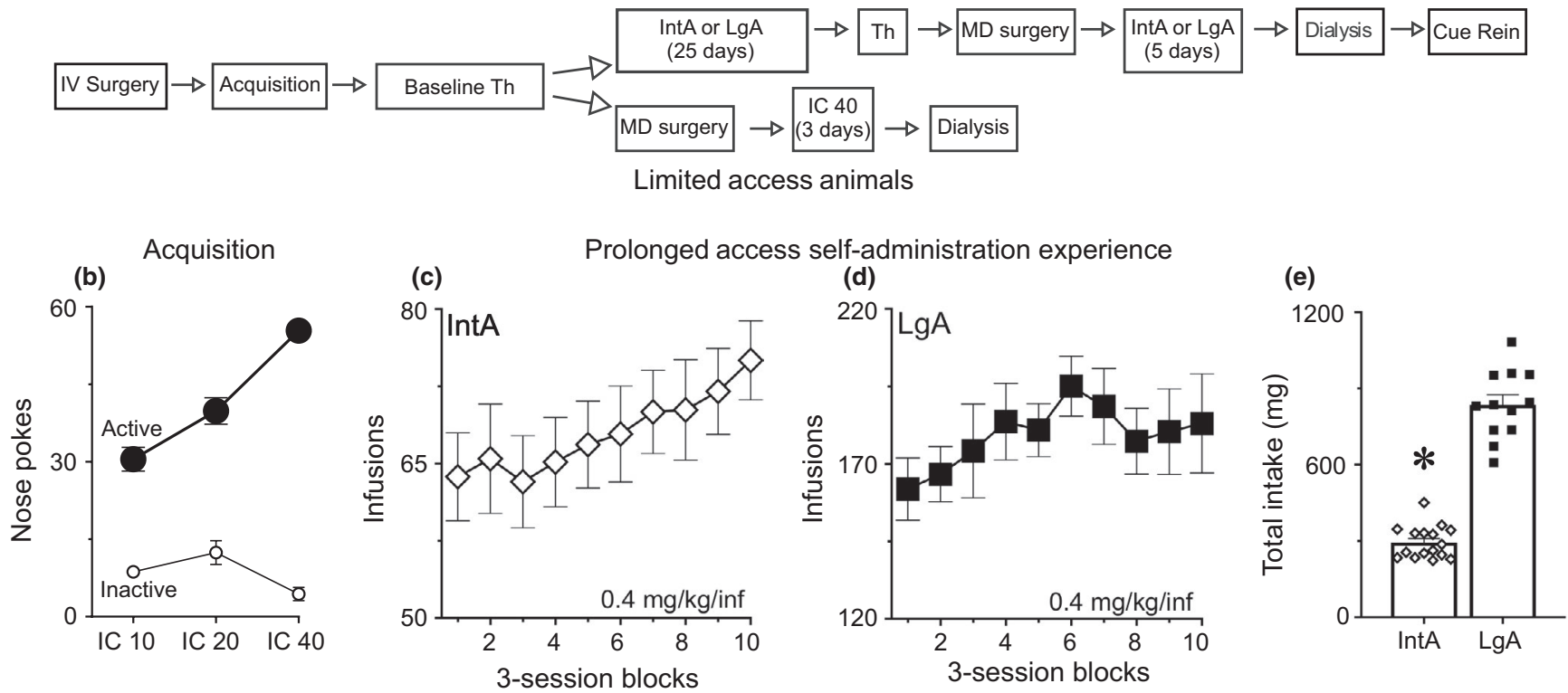

F I G URE 1 The flow diagram shows the overall experimental design and timeline for the experiment (a) (see methods for details). An infusion criterion (IC) procedure was used for the acquisition of cocaine self-administration (b). Both IntA rats and LgA rats escalated their intake/ session to a similar extent across all 30 self-administration sessions (c, d). LgA rats consumed far more cocaine on average than IntA rats over the course of 30 self-administration sessions (e). In panels c and d, each point represents the average of three consecutive self-administration sessions. See methods for the number of rats included in each group and panel. Values represent means \pm SEMs, *represents a significant difference $(p<0.05)$ between IntA and LgA groups (IntA: intermittent access, IV/MD surgery: intravenous catheter/microdialysis guide cannula surgery, LgA: long access, Th: threshold test) 
the LimA group had the same amount of training during acquisition and the threshold test, plus three additional days at IC40, but unlike the IntA and $\operatorname{LgA}$ groups, had no further cocaine self-administration experience prior to the dialysis test session - their cocaine self-administration was relatively limited. Due to complications on the microdialysis test day 1 LimA rat had to be excluded.

\section{5 | Self-administration: intermittent access or long access procedures}

After their behavior stabilized on the threshold procedure, the rats in the prolonged access groups were given 30 selfadministration sessions (Figure 1a). These rats were trained using either an IntA procedure similar to that described previously (Kawa et al., 2016; Zimmer et al., 2012) or a LgA procedure, also similar to that described previously (Ahmed \& Koob, 1998). During the IntA procedure, the rats were placed into the chamber with the house light illuminated. The beginning of the first 5-min Drug-Available period started $2 \mathrm{~min}$ after the rat was placed into the chamber and was signaled by extinguishing the house light. During the Drug-Available period, a nose poke into the active port resulted in an intravenous infusion of cocaine hydrochloride (NIDA) as during acquisition. Each infusion was paired with the illumination of a cue light in the nose port for the duration of the infusion. However, unlike during acquisition, rats could take another infusion immediately after the preceding one (i.e., the only time out period was during the $2.6 \mathrm{~s}$ infusion). After the 5-min drug-available period, the house light turned on and signaled a 25-min no-drug available period. Nose pokes during the no-drug available period were recorded but had no consequence. After 25-min, the house light was extinguished and another 5-min Drug-Available period began. Each IntA session lasted $4 \mathrm{hr}$ (eight cycles of drug-availability).

During the $\operatorname{LgA}$ procedure, the rats were placed into the chamber with the house light illuminated. After $2 \mathrm{~min}$, the house light was extinguished and the rats were able to self-administer cocaine. Each LgA session lasted $6 \mathrm{hr}$ and throughout the session, a nose poke into the active port resulted in an intravenous infusion of cocaine hydrochloride (NIDA) on a FR-1 schedule, as during acquisition. Each infusion was paired with the illumination of a cue light in the nose port for $20 \mathrm{~s}$. Nose pokes during this time were recorded but had no consequences. With the exception of this timeout period, cocaine was available to the rats throughout the 6-hr session. An inactive port was also present at all times and pokes there had no consequences.

Rats in both groups underwent one self-administration session/day for an average of 5 days/week. We varied the number and pattern of days off each week to model the intermittency between bouts of drug use seen in human cocaine users (Cohen \& Sas, 1994; Simon et al., 2002)—for example, one week the rats may have had only 1 day off and then the next week the rats may have had 3 days off. However, rats were never given the day directly before a probe test off. The rats were given 25 self-administration sessions and then were tested again using the within-session threshold procedure. This probe test was identical to the baseline threshold test except that the rats were only tested for 2 days, and the respective values were averaged across the two test days. Two rats in the IntA group were removed from testing prior to this threshold test due to loss of catheter patency. After two threshold test sessions, the rats underwent surgery to implant a microdialysis guide cannula above the nucleus accumbens core, as described below. After 3 days of recovery, the rats were given five additional IntA or LgA self-administration sessions. Also, each rat was first habituated to the microdialysis chamber in which they would be tested for $\sim 1 \mathrm{hr}$ and then at least one of the five additional self-administration sessions was conducted in the microdialysis chamber. Thus, each rat had at least one habituation session and one self-administration session in the microdialysis chamber. Then 1-3 days after the last self-administration session, these rats underwent a microdialysis test and collection session identical to the LimA rats and described below. Due to complications during surgery or the microdialysis test session, $2 \mathrm{Int} A$ rats and $3 \mathrm{LgA}$ rats were excluded, leaving 16 and 9 successful collections, respectively.

\subsection{Extinction and cue-induced reinstatement test}

Following the microdialysis test session, a subset of the Prolonged Access rats was tested for the ability of the cocaine-paired discrete cue (light in the nose port) to reinstate drug-seeking ( $n=10 \mathrm{IntA} ; 9 \mathrm{LgA})$. The rats underwent 2-hr extinction sessions (1/day) for at least 5 days and until they made $<20$ active nose pokes for two consecutive sessions. The extinction sessions consisted of placing the rats into a chamber with the house light on and the session started $2 \mathrm{~min}$ later. Upon the session starting, the house light turned off and remained off for the duration of the session. Responses into the nose ports during these sessions were recorded but had no consequences. The day after a rat met the extinction criterion it underwent a day of testing identical to extinction except on this day pokes in the active port resulted in the illumination of the cue light for 2.6-sec. After the cue-induced reinstatement test, these rats were perfused to verify cannula placement.

\section{7 | Microdialysis}

\subsection{1 | Intracranial placement of cannula for microdialysis}

After their behavior stabilized on the baseline threshold procedure (LimA group) or after 25 additional self-administration 
sessions (IntA and LgA groups), the rats underwent surgery to implant a microdialysis guide cannula above the nucleus accumbens core. Rats were anesthetized with a ketamine xylazine cocktail as described for the catheter implantation and placed in a stereotaxic instrument (David Kopf Instruments, Tujunga, CA). A guide cannula (CMA, CMA12 Guide Cannula) was lowered such that its tip was just above the nucleus accumbens core. The coordinates were $+1.6 \mathrm{~mm}$ anterior, $\pm 1.6 \mathrm{~mm}$ lateral, and $-6.2 \mathrm{~mm}$ ventral, relative to bregma (Paxinos \& Watson, 2007). Hemisphere (right/left) was counter-balanced across all groups. To prevent clogging, a stainless steel stylet was inserted into the cannula. The guide cannula was secured to the skull using three stainless steel screws and acrylic dental cement. The rats were administered the analgesic carprofen $(5 \mathrm{mg} / \mathrm{kg}$ s.c.) once immediately prior to surgery and once each day on the first and second days post-surgery. Rats were allowed at least 3 days of recovery before any subsequent testing.

\subsection{2 | Probe construction and test session}

The microdialysis probes were custom made similar to those described previously (Pitchers, Kane, Kim, Robinson, \& Sarter, 2017). Briefly, two silica capillaries (75 $\mu \mathrm{m}$ inner diameter; $150 \mu \mathrm{m}$ outer diameter; TSP075150; Polymicro Technologies) were glued together and inserted into a 24gauge stainless steel tube that served as the shaft to be inserted into the guide cannula. The portion of each capillary tube that was not inserted into the shaft was sheathed in 22gauge stainless steel tubing and one was used for the inlet capillary and one for the outlet capillary. The capillary tip extending from the shaft was sheathed in an $18 \mathrm{kDa}$ molecular weight cutoff regenerated cellulose membrane (Spectrum Labs). The tip and base of the membrane was sealed with an epoxy resin. The membrane extended $2 \mathrm{~mm}$ in length beyond the shaft, such that a $2 \mathrm{~mm}$ probe would extend into the center of the accumbens core.

In all groups, the microdialysis test session was conducted 1-3 days after the last self-administration session. Approximately $12-16 \mathrm{hr}$ before the microdialysis test session, the stylet was removed from the guide cannula and a probe was inserted. The rat was placed into the microdialysis test chamber with the house light on. The probe was perfused at a rate of $0.5 \mu 1 / \mathrm{min}$ with artificial cerebrospinal fluid (aCSF) overnight and the rate was increased to $1 \mu \mathrm{l} /$ min approximately $4 \mathrm{hr}$ prior to collection. The aCSF was comprised of $145 \mathrm{mM} \mathrm{NaCl}, 2.7 \mathrm{mM} \mathrm{KCl}, 1.0 \mathrm{mM} \mathrm{MgSO}_{4}$, $1.2 \mathrm{mM} \mathrm{CaCl}_{2}, 1.55 \mathrm{mM} \mathrm{NaHPO}_{4}$, and $0.45 \mathrm{mM} \mathrm{NaH}_{2} \mathrm{PO}_{4}$. In addition, $100 \mathrm{nM}^{13} \mathrm{C}_{6}$-DA was added to the aCSF which allowed for in vivo calibration of the probes (Hershey \& Kennedy, 2013) in all but a small subset of the rats (3 LimA, 4 IntA). In this latter subset of rats, recovery rates for each probe were calculated ex vivo by placing the membrane in known concentrations of DA and correcting for the concentration collected in the dialysate sample. These rats did not differ on any measures from rats tested with ${ }^{13} \mathrm{C}_{6}$-DA added to the aCSF and thus were combined for analysis. Approximately $4 \mathrm{hr}$ prior to collection, the rats were also attached to the drug delivery tubing. Dialysate samples were collected every 3 min.

The test session and collection started with 10 baseline samples $(30 \mathrm{~min})$. After $30 \mathrm{~min}$, the house light turned off which signaled that cocaine was available, as it had during all of the previous self-administration sessions. No samples were collected until the rat had made one response in the active nose port and self-administered a single $1.25 \mathrm{mg} / \mathrm{kg}$ cocaine infusion. The light cue that had been paired with cocaine infusions during self-administration testing was not presented, to better isolate the effect of cocaine alone. As soon as the rat made a response and the infusion ended the house light turned back on and no additional cocaine was available to the rat. After accounting for the time it took for the dialysate to travel from the brain to the outlet collection, dialysis samples were collected for $1 \mathrm{hr}$ (20 samples) that corresponded to the onset of the cocaine infusion. One hour after the cocaine infusion, the house light again turned off and the light in the active nose port that had previously been a conditioned stimulus paired with cocaine delivery during self-administration was flashed 14 times for $2.6 \mathrm{~s}$ per flash over a 3 -min period. We collected a single sample that corresponded to this 3-min cue-presentation and five more samples following the cue presentation. Nose pokes in both the active and inactive nose ports were recorded throughout the test session. In summary, 3-min samples were collected over a 30-min baseline period, for 1-hr following a cocaine infusion, for 3 min during which the cue was presented non-contingently, and then for $15 \mathrm{~min}$ following cue presentation.

\subsection{3 | Analysis of neurochemical levels in dialysate samples using high performance liquid chromatography coupled with mass spectrometry}

All reagents, drugs, and chemicals were purchased from Sigma-Aldrich (St. Louis, MO) unless otherwise noted. Samples were analyzed using benzoyl chloride derivatization and a modified LC-MS method previously described (Song, Mabrouk, Hershey, \& Kennedy, 2012). Briefly, the $3 \mu \mathrm{l}$ samples were derivatized by adding $1.5 \mu \mathrm{l}$ of $100 \mathrm{mM}$ sodium carbonate monohydrate buffer, $1.5 \mu \mathrm{l}$ of $2 \%$ benzoyl chloride in acetonitrile, and $1.5 \mu \mathrm{l}$ of an internal standard mixture (to improve quantification), in order, briefly vortexing between each addition. A Thermo Fisher (Waltham, MA) Vanquish UHPLC system automatically injected $5 \mu \mathrm{l}$ of the sample onto a Phenomenex Kinetex C18 HPLC column $(2.1 \mathrm{~mm} \times 100 \mathrm{~mm}, 1.7 \mu \mathrm{m})$. Mobile phase A consisted of 
$10 \mathrm{mM}$ ammonium formate and $0.15 \%$ formic acid. Mobile phase B was pure acetonitrile. Analytes were detected using positive electrospray ionization with a Thermo Fisher TSQ Quantum Ultra triple-quadrupole mass spectrometer operating in multiple-reaction monitoring mode.

Inclusion of ${ }^{13} \mathrm{C}_{6} \mathrm{DA}$ in the aCSF perfusate allowed us to calculate an extraction fraction $\left(E_{\mathrm{d}}\right)$ for each sample (Hershey $\&$ Kennedy, 2013). The $E_{\mathrm{d}}\left(E_{\mathrm{d}}=1-\left(C_{\mathrm{in}} / C_{\text {out }}\right)\right.$ is the ratio of the amount of the isotope that exits the probe to the amount retained in the dialysate sample. Absolute extracellular concentrations of DA were determined by dividing the dialysate concentration of DA by the $E_{\mathrm{d}}$ value. Finally, in a subset of rats we switched the perfusate to aCSF that lacked ${ }^{13} \mathrm{C}_{6}$ DA at the conclusion of the test session. After allowing this aCSF to run for $\sim 1 \mathrm{hr}$, we collected several samples in the same manner as the samples collected during the test. We analyzed these samples and compared them to samples from the baseline portion of the test session. This analysis, along with comparisons between rats that did not have ${ }^{13} \mathrm{C}_{6}$-DA added to the aCSF at any point, confirmed that there were no effects of infusing $100 \mathrm{nM}^{13} \mathrm{C}_{6}$-DA on endogenous DA levels (Figure 3a).

\subsection{Histological analysis}

After the microdialysis test session, rats were anesthetized using sodium pentobarbital $(270 \mathrm{mg} / \mathrm{kg}$; i.p.) and perfused intracardially with $50 \mathrm{ml}$ of $0.9 \%$ saline, followed by $500 \mathrm{ml}$ of $4 \%$ paraformaldehyde in $0.1 \mathrm{M}$ phosphate buffer (PB). After being perfused, brains were removed, post-fixed in the same paraformaldehyde solution for $2 \mathrm{hr}$, then immersed in $20 \%$ sucrose and $0.01 \%$ sodium azide in $0.1 \mathrm{M}$ PB for $48 \mathrm{hr}$ at $4^{\circ} \mathrm{C}$. Coronal sections $(40 \mu \mathrm{m})$ were cut with a freezing microtome (SM 2000R; Leica), collected in PB, and mounted on to a slide immediately. Sections were imaged at $4 \times$ magnification using a Leica DM400B digital microscope to verify cannula placement.

\section{9 $\mid$ Statistical analysis}

Linear mixed-models (LMM) analyses were used for all behavioral repeated measures data. The best-fitting model of repeated measures covariance was determined by the lowest Akaike information criterion score (West, Welch, \& Galecki, 2007). Depending on the model selected, the degrees of freedom may have been adjusted to a non-integer value. Data for the $\alpha$ measure were not normally distributed and therefore all statistical tests involving $\alpha$ were run on $\log$ transformed data, consistent with previous reports (Bentzley, Jhou, \& AstonJones, 2014). Planned post hoc contrasts (and Bonferroni corrections) were done to compare between the different selfadministration procedures and within the Prolonged Access groups across the two test periods. Active and inactive nose pokes from the cue-induced reinstatement test were compared to the last day of extinction and between the IntA and LgA groups. Similar LMM analysis and planned post hoc analysis were used to analyze neurochemical levels from the microdialysis test session. Statistical significance was set at $p<0.05$.

\section{3 | RESULTS}

\section{1 $\quad \operatorname{LgA}$ resulted in much greater cocaine consumption than IntA, but rats in both groups escalated their intake to a similar extent}

The overall timeline of the experiment is shown in Figure 1(a). Figure 1(b) shows the acquisition of cocaine self-administration as a function of Infusion Criterion for all rats, prior to group assignment. Across sessions, the number of responses in the active nose port increased (effect of IC, $F(2,40.4)=38.7, p<0.001)$, and the number of responses in the inactive nose port decreased (effect of IC, $\mathrm{F}(2,75.1)=3.5$, $p=0.04)$. After group assignments, there were no group differences on any measure of acquisition.

A subset of rats was given 30 self-administration sessions using either the IntA or LgA procedure. Rats in the Int A group quickly learned to discriminate between the drug-available and no-drug periods, as previously reported (data not shown; see Kawa et al., 2016). When cocaine intake per session from the IntA and $\operatorname{LgA}$ rats was analyzed together, there was a main effect of test session $(F[29,70.7]=4.8, p<0.001)$. Further analysis confirmed that when each group was analyzed separately both IntA ( $p=0.01$, Figure 1 c) and LgA ( $p=0.02$; Figure 1d), rats escalated their intake as a function of increasing self-administration experience. Relatedly, the rate at which consumption increased did not differ between the groups (group $\times$ session interaction, $F[29,70.7]=1.2$, $p=0.24$ ). There was, however, a large group difference in the total amount of cocaine consumed (effect of group, $F[1,92.3]=823, p<0.001$; Figure 1e). On average, $\mathrm{LgA}$ rats consumed $\sim 2.8 \times$ more cocaine than IntA rats.

\section{2 $\quad$ Prolonged IntA and $\operatorname{LgA}$ experience had different effects on motivated behavior}

All rats underwent a baseline threshold test to quantify their motivation to self-administer cocaine following only limited self-administration experience (Figure 2). Values for each behavioral economic metric were averaged from the last 3 days of the baseline threshold test. Within each measure, there were no differences across the 3 days nor did the variability differ across days. Group assignments (LimA, IntA, $\mathrm{LgA}$ ) were made following this test, such that there were no differences between the groups on any of the measures derived from this test. The rats that were placed into the IntA 
Motivation following IntA and LgA

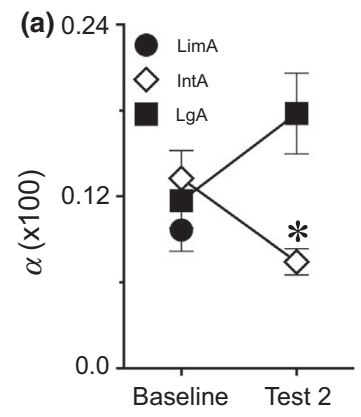

(d)

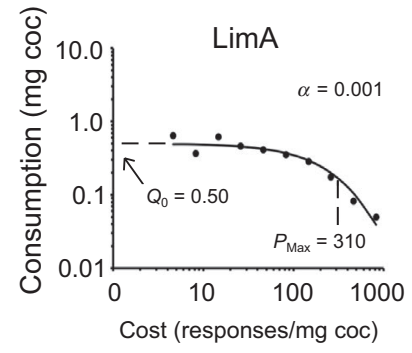

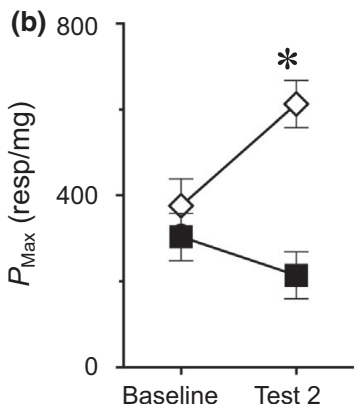

(e)

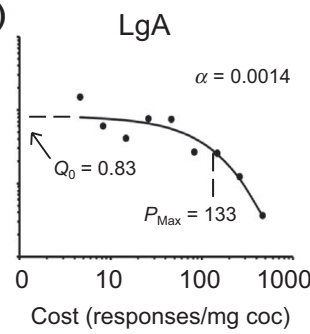

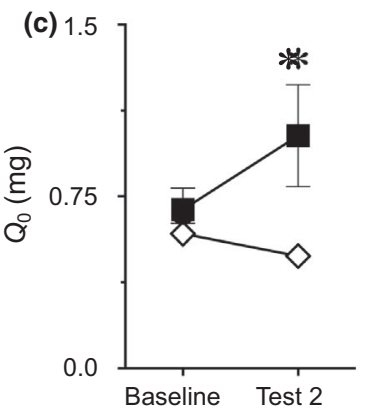

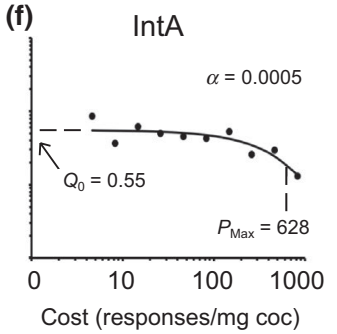

F I G URE 2 Cocaine demand was assessed using a threshold procedure in rats with different self-administration experience. In panels a-c, "baseline" measures are shown for all three groups $(n=42)$ and a subset of rats went on to experience intermittent access (IntA) self-administration $(n=18)$ while a separate group of rats went on to long access ( $\mathrm{LgA})$ self-administration $(n=12)$. Group assignments were made to insure there were no group differences on these measures at baseline. Baseline $\alpha$ values for all three groups are shown in panel (a), which also shows that IntA experience decreased $\alpha$ (increased motivation) relative to LgA experience (a). Similarly, IntA, but not LgA, increased $P_{\text {Max }}$ (increased motivation) (b). $\mathrm{LgA}$ experience increased $Q_{0}$ (preferred cocaine intake when effort is not a factor) relative to IntA experience (c). Panels d-f show representative demand curves from a rat after LimA experience (d), prolonged IntA (e), and prolonged $\operatorname{LgA}(\mathrm{f})$. In panels b and c, baseline values from the LimA group are plotted but difficult to see as they overlap with the baseline LgA values. Values represent means \pm SEMs, $\alpha$ values shown in (a) are calculated values multiplied by $100,{ }^{*}$ represents a significant difference $(p<0.05)$ between IntA and LgA groups

and $\operatorname{LgA}$ groups then underwent 25 self-administration sessions before another threshold probe test to determine how motivation changed as a function of IntA or LgA experience, relative to baseline. For this second threshold test, values for each behavioral economic metric were averaged from the two test days. Within each measure, there were no differences across the 2 days nor did the variability differ across days. All statistical comparisons to baseline were within-subject.

The metric $\alpha$ is a measure of the elasticity of the demand curve generated during the within-session threshold procedure, and is inversely proportional to motivation (Bentzley et al., 2013; Figure 2a). Prolonged IntA and $\operatorname{LgA}$ experience had different effects on $\alpha$, as indicated by a significant group $\times$ probe test interaction $(F[1,69]=7.2, p=0.009)$. Post hoc within-subject analysis revealed that IntA experience decreased $\alpha$ (increased motivation) $(p=0.02)$ whereas LgA experience did not significantly change $\alpha(p=0.12)$. On the second probe test, IntA rats had a lower $\alpha$ than $\mathrm{LgA}$ rats (effect of group, $p=0.04$ ), indicating that rats with IntA experience were more motivated to obtain cocaine.

The metric $P_{\mathrm{Max}}$ is a measure of motivation that reflects the maximum price an individual is willing to pay (in effort) to obtain a reinforcer (Bentzley et al., 2013; Figure 2b). Again, prolonged IntA and LgA had different effects on $P_{\text {Max }}$, as indicated by a significant group $\times$ probe test interaction $(F[1,33.1]=10.4, p=0.003)$. Post hoc within-subject analysis revealed that IntA experience increased $P_{\text {Max }}(p=0.001)$, and LgA experience did not significantly change $P_{\mathrm{Max}}$ $(p=0.27)$. On the second probe test, IntA rats had a higher $P_{\text {Max }}$ than $\mathrm{LgA}$ rats (effect of group, $p=0.005$ ), indicating that they were more motivated to obtain cocaine, consistent with $\alpha$.

Another metric derived from the threshold procedure is $Q_{0}$, which measures the preferred level of cocaine consumption when cost is nil (Bentzley et al., 2013; Figure 2c). Again there was a significant group $\times$ probe test interaction $(F[1,28.7]=7.7, p=0.009)$ indicating that IntA and $\operatorname{LgA}$ had different effects on $Q_{0}$. $Q_{0}$ differed from $\alpha$ and $P_{\text {Max }}$ as post hoc within-subject analysis revealed that $\operatorname{LgA}$ experience increased $Q_{0}(p=0.01)$ but IntA experience had no effect on $Q_{0}(p=0.25)$. Finally, $\operatorname{LgA}$ rats had higher $Q_{0}$ than IntA rats after prolonged self-administration (effect of group, $p=0.03)$. These differential effects of $\operatorname{LgA}$ and IntA on $Q_{0}$ are consistent with several reports (Bentzley et al., 2014; James et al., 2018; Kawa et al., 2016; Oleson \& Roberts, 2009; Singer, Fadanelli, Kawa, \& Robinson, 2018).

Representative demand curves generated from the threshold procedure are shown from a LimA rat on the 
baseline threshold test (Figure 2d), and from a LgA rat (Figure 2e) and an IntA rat (Figure 2f) on the second threshold test.

\section{3 | Infusion of stable-isotope labeled dopamine did not affect endogenous dopamine levels}

Use of extraction fraction $\left(E_{\mathrm{d}}\right)$ to calculate in vivo concentration accounts for possible differences in recovery associated with the probe and brain environment in different rats. In vivo calibration therefore improves the accuracy of such measures and allows comparison between rats. $100 \mathrm{nM}$ stable-isotope labeled DA $\left({ }^{13} \mathrm{C}_{6}\right.$-DA) was included in the aCSF that was perfused during the microdialysis test session to calculate an $E_{\mathrm{d}}$ for each sample (see methods; Hershey \& Kennedy, 2013). As a control, we determined if the infusion of this concentration of ${ }^{13} \mathrm{C}_{6}$-DA affected DA levels in dialysate [DA]. For this control measurement, the input solution was switched to aCSF that lacked ${ }^{13} \mathrm{C}_{6}$-DA at the end of each dialysis test session and after allowing the lines to clear of any ${ }^{13} \mathrm{C}_{6}$-DA aCSF three more dialysate samples were collected to compare to the last samples collected with ${ }^{13} \mathrm{C}_{6}$-DA aCSF. As we have reported previously (Hershey \& Kennedy, 2013), the presence of ${ }^{13} \mathrm{C}_{6}$-DA did not affect [DA] (Figure 3a). In addition, the calculated $\mathrm{E}_{\mathrm{d}}$ did not differ between groups nor did they change across the dialysis test session (all $p$-values $>0.1$; Figure $3 b$ ). These results provide confidence that the calibrated [DA] concentrations reported can be compared between rats and groups.

\section{4 | Prolonged IntA (but not LgA) sensitized cocaine-induced dopamine overflow}

Following the collection of baseline dialysate for $30 \mathrm{~min}$, rats with LimA, prolonged IntA, or prolonged LgA experience were allowed to self-administer a single IV injection of $1.25 \mathrm{mg} / \mathrm{kg}$ cocaine (see Figure $4 \mathrm{a}$ for timeline), and dialysate was then collected for an additional $60 \mathrm{~min}$. There were no group differences in the latency to self-administer the cocaine infusion as most rats nose poked as soon as the house light turned off signaling drug availability. We first analyzed the average concentration of [DA] in the 10 baseline samples and the first 10 post-cocaine samples in all three groups (Figure 4b). When analyzing all 20 samples, there was a main effect of group $(F[2,326]=10.2$, $p<0.001)$ and the cocaine infusion increased [DA], relative to baseline, in all groups (main effect of cocaine, $F[1,331]=8.93, p=0.003$; Figure $4 \mathrm{~b}$ ). Planned post hoc analysis revealed that the main effect of group was due to a differential response to the cocaine infusion. That is, there were no group differences in baseline [DA] (all $p$-values $>0.1$ ) but following the cocaine infusion, $[\mathrm{DA}]$ increased to
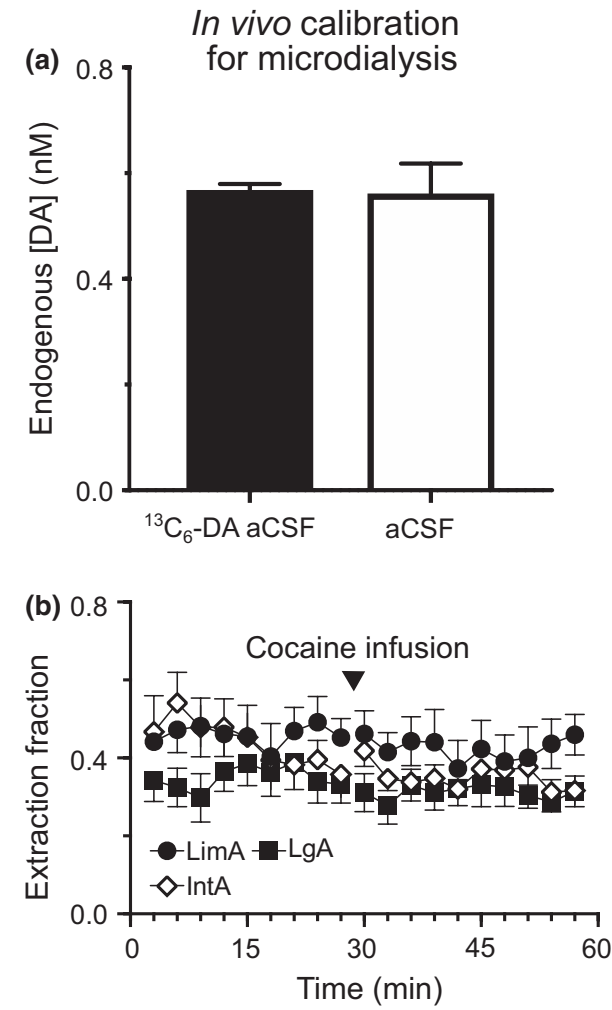

F I G U RE 3 Stable-isotope labeled DA $\left({ }^{13} \mathrm{C}_{6}\right.$-DA $)$ was included in the artificial cerebrospinal fluid (aCSF) perfusate during the dialysis test session in order to calculate an extraction fraction $\left(E_{\mathrm{d}}\right)$ for each sample collected. Infusing ${ }^{13} \mathrm{C}_{6}$-DA did not affect endogenous dopamine concentration in dialysate [DA] (here not corrected for probe recovery) when compared to aCSF that lacked ${ }^{13} \mathrm{C}_{6}$-DA (a). The average calculated $E_{d}$ throughout the test did not differ between LimA, LgA, or IntA groups (10 baseline and 10 post-cocaine samples shown) (b). Values represent means \pm SEMs

a greater extent in IntA rats, relative to $\operatorname{LgA}$ rats $(p<0.001)$ and LimA rats $(p=0.004)$. The latter two groups did not differ $(p=0.21)$. The largest [DA] response to cocaine reliably occurred within the first three samples $(9 \mathrm{~min})$ after the infusion, but there was individual variation in the time that elapsed from infusion to peak response. To better visualize the peak response to cocaine in all individuals, Figure 4(c) shows $[\mathrm{DA}]$ in all three groups when each rat's peak response was aligned.

Given that there were no group differences in baseline [DA], we averaged the baseline values together for each group to determine the percent change from baseline produced by cocaine (Figure 4d). For this analysis, we aligned each rat's peak [DA] response. There was a main effect of group $(F[2,672]=6.04$, $p=0.003)$ and the cocaine infusion increased [DA] in all groups $(F[1,673]=40, p<0.001)$. Further, the cocaine infusion increased $[\mathrm{DA}]$ to a different extent in the three groups evidenced by a group $\times$ cocaine interaction $(F[2,672]=6.04$, $p=0.003$ ). Planned post hoc analysis revealed that the cocaine infusion increased $[\mathrm{DA}]$ to a greater extent in IntA rats than 
(a)
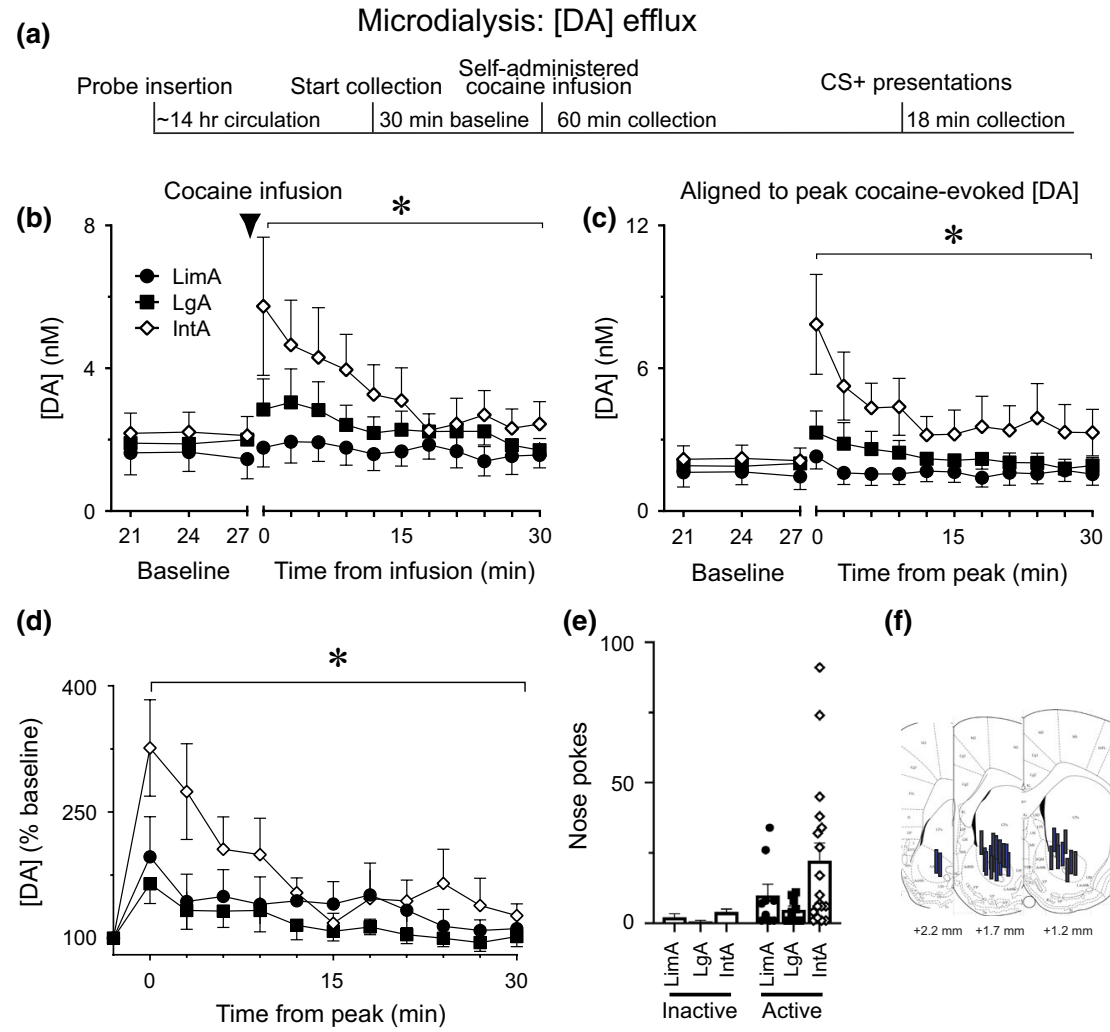

(f)

F I G U RE 4 [DA] in response to a self-administered cocaine infusion was tested in rats with different self-administration experience. A timeline of the microdialysis test day is shown in panel (a). Data from the conditioned stimulus (CS presentation) are not shown as there was no change in [DA]. Cocaine-evoked [DA] was greater in rats trained with IntA $(n=16)$ than rats trained with $\operatorname{LgA}(n=9)$ or $\operatorname{LimA}(n=9)(\mathrm{b})$. Panel $\mathrm{c}$ shows the same data with each rat's peak response to cocaine aligned. When peak [DA] was analyzed as a percent of baseline, cocaine evoked a greater increase in [DA] in IntA rats than LgA or LimA rats (d). The number of active nose pokes made in the 60 min following the cocaine infusion is shown in panel e. Microdialysis probes were targeted to the nucleus accumbens core (f). Values represent means \pm SEMs, * represents a significant difference $(p<0.05)$ between groups. [Colour figure can be viewed at wileyonlinelibrary.com]

LgA rats $(p<0.001)$ or LimA rats $(p=0.016)$, and the latter two groups did not differ $(p=0.1)$, consistent with the analysis of [DA] that was not normalized to baseline.

Active nose pokes that were made in the hour following the single self-administered cocaine infusion during the microdialysis test session were recorded and analyzed as a measure of cocaine-induced drug seeking. A one-way ANOVA comparing the three groups revealed no effect of group on nose pokes, although there was a trend toward IntA rats making more responses than LimA or LgA rats (effect of group, $F[2]=2.7, p=0.08$; Figure 4e).

\section{5 | IntA rats showed greater cue-induced reinstatement than $\operatorname{LgA}$ rats}

The day after the microdialysis test session, a subset of the rats in the IntA group and the $\operatorname{LgA}$ groups underwent extinction training followed by a test for cue-induced reinstatement of cocaine-seeking (conditioned reinforcement). There were no group differences during extinction training in the number of responses made or the number of sessions required to reach extinction criteria (all $p$-values $>0.1$; Figure $5 \mathrm{a}$ ). On the test day, when responding was reinforced by the presentation of the cue that had previously been paired with cocaine, both IntA and $\mathrm{LgA}$ rats reinstated their drug-seeking relative to extinction levels $(F[1,20.1]=100, p<0.001$; Figure $5 b)$, specifically at the active nose port (effect of inactive nose port $\times$ session, $F[1,18.1]=2.3, p=0.15$ ). However, rats with IntA experience showed more robust cue-induced reinstatement than rats with $\mathrm{LgA}$ experience (effect of group, $F[1,20.1]=22, p<0.001)$.

\section{6 | Cocaine-seeking on multiple tests of addiction-like behavior predicted cocaine- evoked dopamine overflow}

Given the role of DA in motivated behavior, we asked whether [DA] in response to self-administered cocaine correlated with cocaine-seeking behavior and/or motivation for cocaine. Figure 6 shows that the magnitude of the increase in $[\mathrm{DA}]$, as a percent of baseline, on the microdialysis test day was positively correlated with cocaine-seeking 


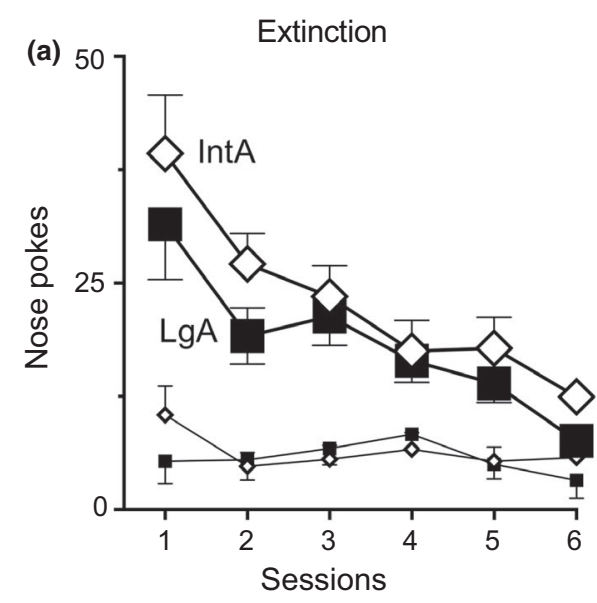

(b) Cue-induced reinstatement

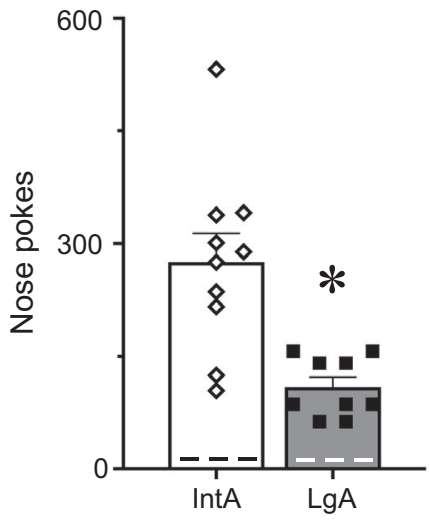

F I G URE 5 After the dialysis test session, a subset of intermittent access (IntA) rats $(n=10)$ and long access $(\mathrm{LgA})$ rats $(n=9)$ were tested for the ability of a cocaine-paired cue to reinstate drug-seeking. IntA and $\operatorname{LgA}$ rats did not differ in responding during extinction training (a). In a test for conditioned reinforcement, when a nose poke in the previously active port was reinforced by presentation of the cue that had previously been associated with cocaine but no cocaine, IntA rats made more responses than LgA rats (b). Nose pokes at the inactive port are represented by the smaller lines in panel a and dashed lines in panel b. Values represent means \pm SEMs, * represents a significant difference $(p<0.05)$ between IntA and LgA groups

on the dialysis test day, assessed by the number of nose pokes into the active port $\left(R^{2}=0.14, p=0.03\right.$; Figure 6a). There was a similar correlation between cocaine-induced [DA] and motivation for cocaine $\left(P_{\mathrm{Max}}\right)$ assessed on the most recent threshold test (baseline test for LimA; second test for IntA/LgA) $\left(R^{2}=0.32, p<0.001\right.$; Figure 6b). This correlation was evident even when the analysis was restricted to the IntA group $\left(R^{2}=0.29, p=0.03\right)$. There was a similar correlation between [DA] and $\alpha\left(R^{2}=0.13\right.$, $p=0.04$; data not shown). In contrast, $Q_{0}$ did not correlate with [DA] when all groups were combined $\left(R^{2}=0.07\right.$, $p=0.17$; Figure $6 \mathrm{c}$ ) or within any individual group (all $p$-values $>0.1)$.

Int A experience reliably produces multiple addictionlike behaviors and this has been shown to be particularly
Addiction-like behaviors and DA release

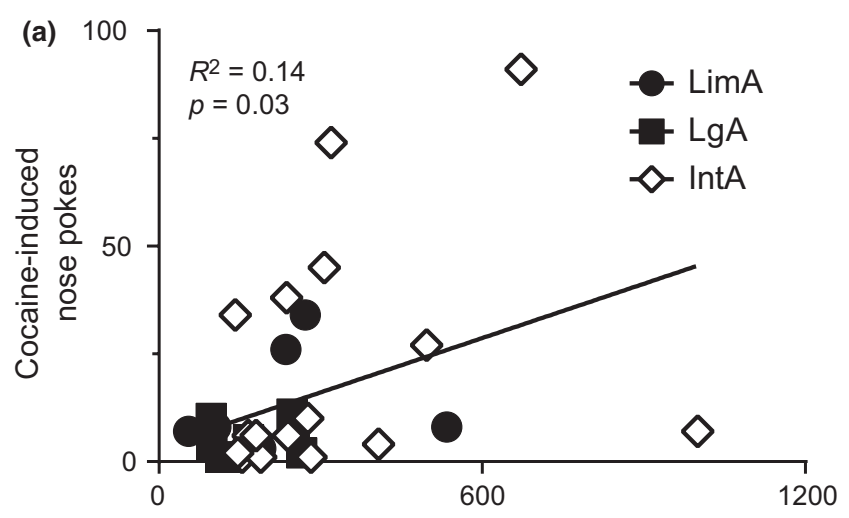

(b)

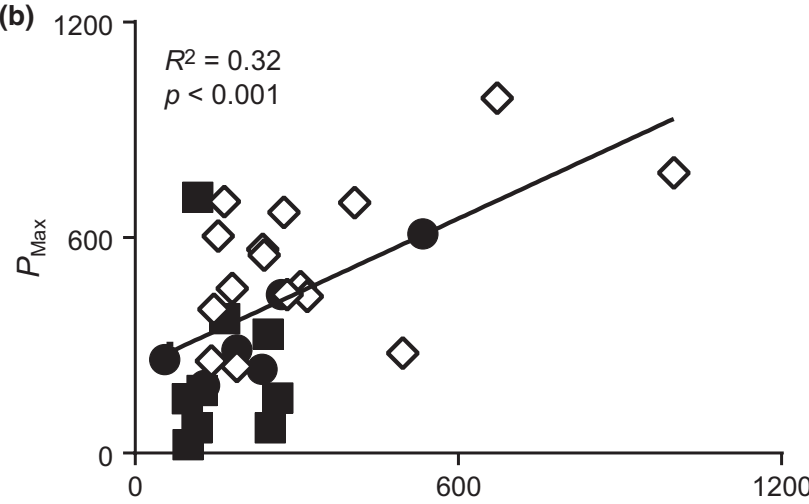

(c)

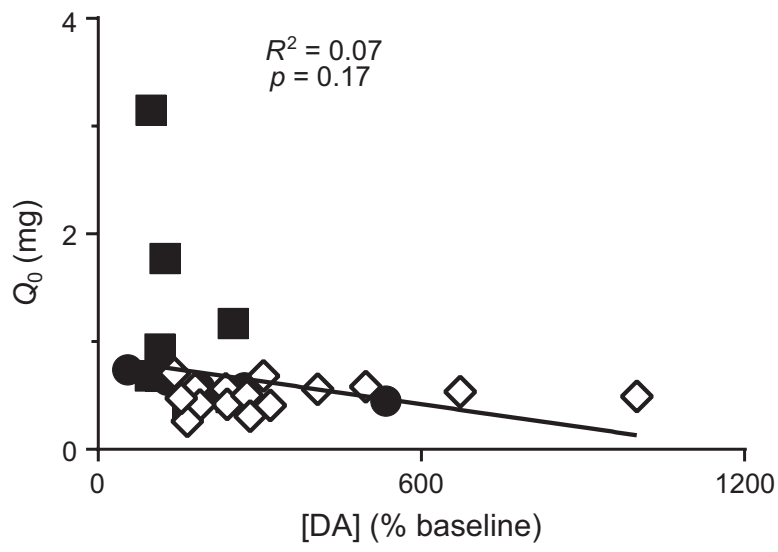

F I G URE 6 Each rat's peak [DA] response to a self-administered cocaine infusion, as a percent of baseline, was correlated with that rat's performance on several tests of addiction-like behavior. The peak [DA] response, as a percent of baseline, predicted cocaine-induced nose pokes (measured during the microdialysis test session) (a) and $P_{\mathrm{Max}}$ from the most recent threshold test (b), but did not predict $Q_{0}$ from the most recent threshold test (c)

robust in susceptible individuals (Kawa et al., 2016; Singer et al., 2018). In an effort to quantify this variation in behavior, we developed an "IntA Addiction Score", similar to the oft-used addiction-criteria analysis (Deroche-Gamonet et al., 2004; Kawa et al., 2016; Singer et al., 2018), but differing in the behaviors that were analyzed. Rats that were trained with the IntA procedure 
were separated into those that had a score of $2 / 3$ ("High") and those that had a score of 0/1 ("Low"). Briefly, a rat was given an "Addiction Score" point if it was within the top third of the population on a given measure. The measures used here were $\alpha$, active nose pokes during the no-drug period of IntA, and active cocaine-induced nose pokes during the microdialysis test session (Figure 7; see discussion for rationale of using these measures). There were nine rats that scored 0/1 points ("Low") and seven rats that scored 2/3 points ("High"). Not surprisingly, the low and high groups differed in their performance on the behavioral measures for which they were classified (Figure $7 \mathrm{a}-\mathrm{c}$ ). In addition, the low and high groups differed in $P_{\text {Max }}$ but did not differ in $Q_{0}$ (data not shown). Importantly, high rats showed greater cocaine-evoked [DA] than low rats $(p=0.05$; Figure $7 d)$. Finally, when High IntA rats (the most "addict-like") were compared to LimA and $\operatorname{LgA}$ rats (that largely did not show addiction-like behavior), the cocaine infusion increased [DA] to a greater extent in High IntA rats (group $\times$ cocaine interaction, $F[2,484]=19.5, p<0.001$; Figure $7 \mathrm{e}$ ).

\section{7 | Self-administered cocaine increased extracellular glutamate and 3-MT levels}

The high performance liquid chromatography coupled with mass spectrometry (HPLC-MS) technique used here allows for the analysis of a number of analytes, in addition to DA (Table 1). There were no group differences in the baseline levels of any of the analytes quantified, although there was a trend toward group differences in baseline glutamate levels (effect of group, $F[2,328]=2.7, p=0.08$; post hoc analysis revealed this to be driven primarily by elevated baseline levels in LimA rats). Therefore, all values were normalized to baseline and cocaine-induced changes relative to baseline were calculated. Self-administered cocaine increased extracellular glutamate levels relative to baseline in all groups (effect of cocaine, $F[1,671]=3.97, p=0.04$ ), but there were no group differences $(p>0.1)$. In addition, self-administered cocaine increased 3-MT, a DA metabolite formed extracellularly, in all groups (effect of cocaine, $F[1,581]=10.5$, $p<0.001)$. Further, cocaine increased 3-MT to a greater extent in IntA rats than $\mathrm{LgA}$ or LimA rats (group $\times$ cocaine

Int $\mathrm{A}$ addiction score analysis
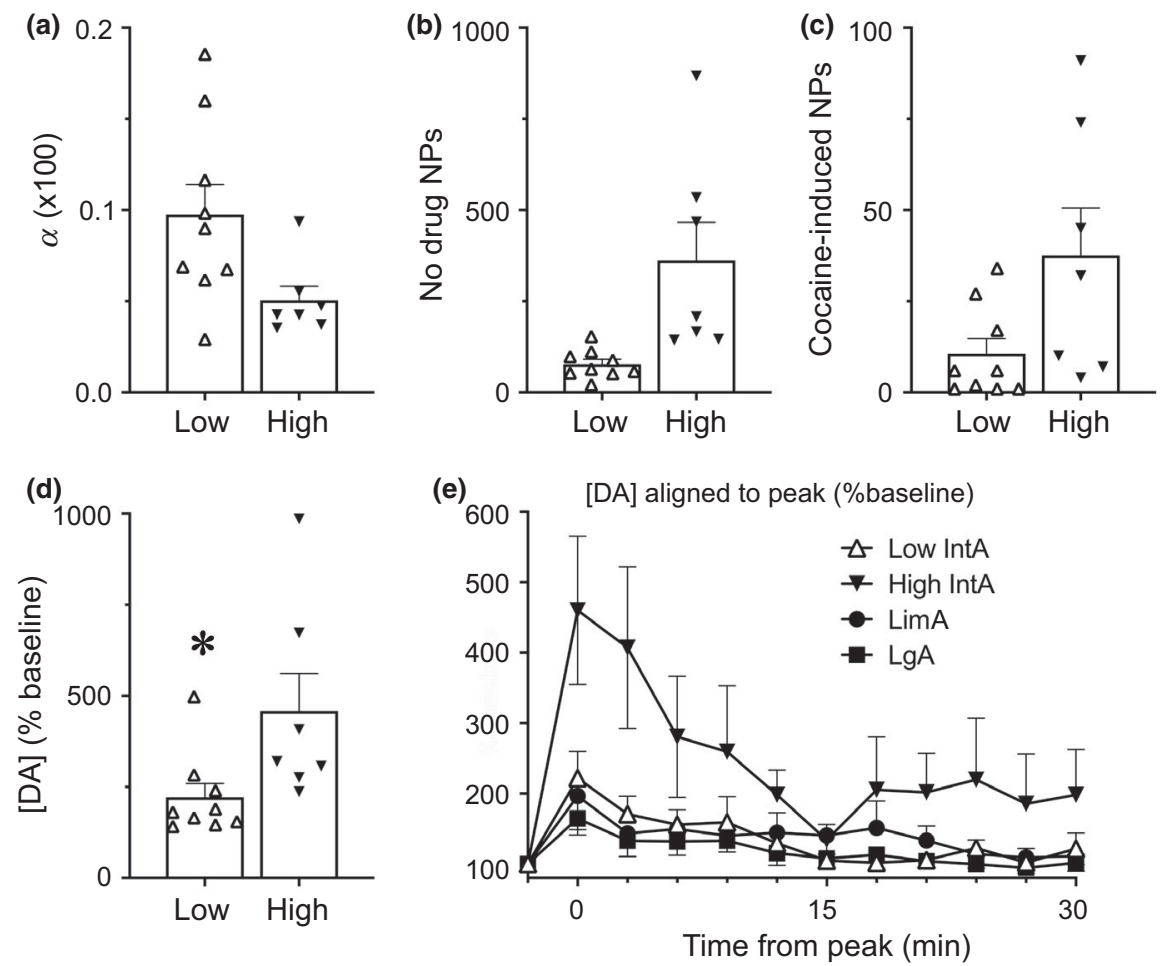

F I G U RE 7 Analysis based on "IntA Addiction Score". Intermittent access (IntA) rats were separated based on their "Addiction Score" (see methods). Rats that scored $2 / 3$ points $(n=7)$ were classified as "High" and showed increased motivation relative to rats that scored $0 / 1$ points $(n=9)$, classified as "Low", as indicated by alpha $(\alpha)($ a), active nose pokes (NPs) made during the no-drug periods of IntA (b), and cocaineinduced active nose pokes (measured during the microdialysis test day) (c). A self-administered infusion of cocaine evoked a greater increase in [DA], shown as a percent of baseline, in "High" rats than "Low" rats (d). Panel e shows the timecourse of [DA] (\% baseline) following cocaine in "High" and "Low" IntA rats, LimA rats, and LgA rats. Values represent means \pm SEMs, $\alpha$ values shown in (a) are calculated values multiplied by 100 , * indicates a significant $(p<0.05)$ difference between groups 
TA BLE 1 Other analytes of interest following a cocaine infusion. Microdialysis allows for the analysis of a number of analytes in addition to DA. Select neurochemicals of interest are shown here. A self-administered cocaine infusion increased extracellular levels of glutamate and 3-MT (a dopamine metabolite) in all rats. Cocaine-evoked 3-MT was greater in IntA rats than $\mathrm{LimA}$ rats or $\mathrm{LgA}$ rats. * indicates a significant $(p<0.05)$ change in analyte concentration following the cocaine infusion relative to baseline

\begin{tabular}{llll} 
Analytes & Group differences & $\begin{array}{l}\text { Peak cocaine-induced } \\
\text { change from baseline }\end{array}$ & SE \\
\hline Glutamate & No & $+32 \% *$ & $\pm 10.3 \%$ \\
GABA & No & $+1.4 \%$ & $\pm 4.6 \%$ \\
\hline ACh & No & $-10 \%$ & $\pm 33 \%$ \\
3 -MT & Yes & IntA: $+98 \% *$ & $\pm 26 \%$ \\
& & Lim\&LgA: $+25 \% *$ & $\pm 9.1 \%$ \\
DOPAC & No & $-9.9 \%$ & $\pm 4.1 \%$ \\
HVA & No & $+0.5 \%$ & $\pm 5.2 \%$ \\
\hline
\end{tabular}

3-MT, 3-methoxytyramine; ACh, acetylcholine; DOPAC, 3,4-dihydroxyphenylacetic acid; GABA, gammaaminobutyric acid; HVA, homovanillic acid. interaction, $F[2,580]=4.93, p=0.008$; post hoc analysis revealed this to be driven by IntA rats). We also analyzed extracellular GABA, ACh, DOPAC, and HVA levels but none of these differed between groups or changed significantly following cocaine.

\section{8 | Presentation of the cocaine- paired cue did not affect extracellular neurochemical levels}

One hour after the self-administered cocaine infusion, the conditioned stimulus that had been paired with cocaine infusions during self-administration training was presented non-contingently for $3 \mathrm{~min}$. Presentation of the conditioned stimulus did not produce any detectable changes in any of the analytes that we measured (data not shown; see discussion).

\section{4 | DISCUSSION}

The purpose of this study was to compare the ability of prolonged $\mathrm{LgA}$ and IntA cocaine self-administration experience to produce addiction-like behavior (relative to LimA), and how this influenced the ability of self-administered cocaine to change the concentration of DA in dialysate $[\mathrm{DA}]$ in the nucleus accumbens core in vivo. The main findings were: (a) as expected, $\mathrm{LgA}$ resulted in much greater total cocaine consumption than IntA. (b) Both IntA and LgA produced escalation of intake with increasing self-administration experience. (c) IntA (but not $\operatorname{LgA}$ ) experience increased motivation for cocaine, as indicated by a decrease in $\alpha$ and increase in $P_{\mathrm{Max}}$. (d) IntA rats showed greater cue-induced reinstatement of cocaine-seeking than LgA rats. (e) LgA (but not IntA) experience increased the preferred level of cocaine intake when no effort was required $\left(Q_{0}\right)$. (f) There were no group differences in the basal levels of [DA], but a single self-administered IV injection of cocaine increased [DA] in the core of the nucleus accumbens to a greater extent in rats with prior IntA experience than those with $\operatorname{LgA}$ or LimA experience, and the latter two groups did not differ. (g) Across all groups, high motivation for cocaine was associated with a greater [DA] response. (h) There were no group differences in dialysate concentrations of glutamate, GABA, ACh, DOPAC or HVA, although cocaine increased 3-MT to a greater extent in IntA than LimA or LgA rats, consistent with the effects on DA.

\section{1 | IntA experience was more effective in producing addiction-like behavior than LgA experience}

Since its introduction in 1998 (Ahmed \& Koob, 1998) the $\mathrm{LgA}$ procedure has been widely adopted to model the transition to cocaine addiction in rats, because it was thought to be especially effective in producing a number of addictionlike behaviors, relative to short or more LimA procedures (for reviews see, Ahmed, 2012; Edwards \& Koob, 2013). In their 1998 paper, Ahmed and Koob reported that LgA, but not shorter access, resulted in escalation of intake. Since that time, it has also been reported that, relative to shorter access, rats with $\operatorname{LgA}$ experience are more motivated to seek cocaine (Paterson \& Markou, 2003; Wee, Mandyam, Lekic, \& Koob, 2008), take more cocaine in the face of adverse consequences (Xue, Steketee, \& Sun, 2012; Bentzley et al., 2014; also see Vanderschuren \& Everitt, 2004), and show greater reinstatement of cocaine-seeking behavior following extinction (Ahmed \& Cador, 2006; Kippin, Fuchs, \& See, 2006; Mantsch, Yuferov, Mathieu-Kia, Ho, \& Kreek, 2004; Mantsch et al., 2008). As indicated by the excerpt cited in the Introduction from Ahmed (2012), it has been suggested that the critical factor necessary for the emergence of escalation and other addiction-like behavior is the amount of drug consumed. As put by Edwards and Koob (2013), "excessive drug exposure likely remains an indispensable element driving the development of addiction". However, the findings presented here add to a growing literature indicating that this is not the case.

IntA self-administration results in much less total cocaine consumption than $\mathrm{LgA}$. Yet as reported here, IntA also 
produced escalation of intake and was more effective than $\mathrm{LgA}$ in increasing motivation for cocaine and in producing cue-induced reinstatement of cocaine-seeking behavior. These findings are consistent with a number of recent studies that also report IntA produces escalation of intake, heightened motivation for cocaine, continued cocaine-seeking in the face of an adverse consequence, continued cocaine-seeking when it is not available, and greater cue-induced reinstatement (Allain \& Samaha, 2018; Allain et al., 2018; James et al., 2018; Kawa \& Robinson, 2019; Kawa et al., 2016; Singer et al., 2018; Zimmer et al., 2012). Collectively, these studies have established that the consumption of the large amount of cocaine associated with $\operatorname{LgA}$ is not necessary for the development of addiction-like behavior and other pharmacokinetic factors appear to be more important (for reviews see Allain et al., 2015; Kawa et al., 2019).

The failure of $\operatorname{LgA}$ experience to increase motivation for cocaine in the present study is inconsistent with several previous studies using either the same behavioral economic indicators used here (Bentzley et al., 2014; Zimmer et al., 2012) or progressive ratio (PR) tests (Paterson \& Markou, 2003; Wee et al., 2008). However, the effects of LgA reported in these studies were often only assessed at a single time point and compared to shorter access, and did not involve withinsubject comparisons. In the studies that measured how motivation changed with increasing $\operatorname{LgA}$ experience (Bentzley et al., 2014), the effects were modest relative to the changes that occur following IntA. Our findings are consistent with other reports that $\mathrm{LgA}$ experience does not increase motivation for cocaine, as assessed with either behavioral economic metrics (Oleson \& Roberts, 2009) or PR tests (Liu, Roberts, \& Morgan, 2005; Quadros \& Miczek, 2009; Willuhn et al., 2014 supplementary). In addition, it has been reported that changes in motivation produced by LgA experience are very transient, lasting for only a few days after the last self-administration session (Bentzley et al., 2014; James et al., 2018), whereas the increased motivation produced by IntA experience is long-lasting — still evident after 50 days of abstinence (James et al., 2018). In summary, evidence that LgA increases motivation for cocaine is somewhat mixed, whereas IntA has been consistently reported to do so.

When allowed to self-administer cocaine under low FR schedules of reinforcement, rats generally titrate their responding to achieve a preferred brain concentration of cocaine, which they defend within a wide range of doses (Ahmed \& Koob, 1999; Gerber \& Wise, 1989; Lynch \& Carroll, 2001). This preferred level of consumption was quantified here by the metric $Q_{0}$-the preferred level of consumption when cost is nil. $Q_{0}$ presumably represents the brain level of cocaine that produces some optimal desired effect, such that neither more nor less cocaine is better. Some have referred to $Q_{0}$ as a "hedonic set-point" (Bentzley et al., 2013), although "settling-point" may be more appropriate (see Berridge,
2004). Of course, it is not possible to know if $Q_{0}$ actually reflects subjective hedonic effects in rodents. Nevertheless, $\operatorname{LgA}$ experience does increase the preferred level of cocaine consumption, as reported here and by others (Bentzley et al., 2014; James et al., 2018; Oleson \& Roberts, 2009). The present results suggest, therefore, that $\operatorname{LgA}$ experience produces tolerance to whatever desired effect of cocaine is defended as price increases, without any change in motivation for cocaine. In contrast, IntA increases motivation for cocaine without any concomitent change in cocaine's desired effects. Although speculative, this may reflect a dissociation between cocaine "wanting" and "liking" (Berridge \& Robinson, 2016; Robinson \& Berridge, 1993). It also suggests that cocaine intake may escalate with both $\operatorname{LgA}$ and IntA, but for very different reasons-because of tolerance to cocaine's desired effect in the case of $\operatorname{LgA}$ and incentive-sensitization in the case of IntA (Kawa \& Robinson, 2019; Kawa et al., 2016). The idea that consummatory and motivational aspects of behavior are psychologically (and neurobiologically) dissociable has been suggested frequently (e.g., Guillem, Ahmed, \& Peoples, 2014; Nicola \& Deadwyler, 2000; Oleson et al., 2011; Sharpe \& Samson, 2001).

\section{2 | Neither LgA nor IntA experience altered basal dopamine}

A decrease in basal DA levels has been reported when testing occurred soon after the discontinuation of high-dose and/ or high-intake cocaine self-administration procedures (Ferris et al., 2011; Mateo, Lack, Morgan, Roberts, \& Jones, 2005). However, in the present study neither LgA nor IntA experience had any effect on basal [DA]. Also, we included ${ }^{13} \mathrm{C}_{6}$ DA in the aCSF, which allowed us to calculate an extraction fraction for each sample, and thus more accurately estimate basal [DA]. There were no group differences in the extraction fraction, thus bolstering our conclusion that neither $\operatorname{LgA}$ nor IntA changed basal [DA] (relative to LimA). This result is consistent with other reports that $\operatorname{LgA}$ experience does not alter baseline [DA], relative to rats with shorter access to cocaine (Ahmed et al., 2003) or drug-naive rats (Calipari et al., 2014). In addition, baseline [DA] did not correlate with any of our measures of addiction-like behavior, consistent with other studies (Ahmed et al., 2003; Hurd, Weiss, Koob, And, \& Ungerstedt, 1989).

\section{3 | IntA, but not $\mathrm{LgA}$, sensitizes cocaine- evoked dopamine overflow}

There have been very few studies on the neurobiological consequences of IntA experience, and those available have all involved ex vivo measures. Most relevant to the present study are reports by (Calipari et al. 2013; Calipari, Siciliano, Zimmer, \& Jones, 2015) that IntA experience sensitizes 
stimulated DA release from the nucleus accumbens core in tissue slices, relative to naive rats or rats with a history of shorter access self-administration, and also increases the ability of cocaine to inhibit DA uptake. The main purpose of the present experiment was to determine if a similar sensitization of DA neurotransmission is present in awake, behaving rats. Following prolonged IntA experience a single self-administered cocaine infusion, given in the absence of the cocaine cue, produced a greater increase in extracellular [DA] in the accumbens core than following either LgA or LimA experience, and these latter two groups did not differ.

Furthermore, the magnitude of the [DA] response predicted motivation for cocaine, as assessed by a number of measures, including $P_{\text {Max }}, \alpha$, and cocaine-seeking on the microdialysis test day. In addition, the [DA] response to cocaine was greatest in rats with IntA experience that scored the highest on an "IntA Addiction Score". The ability of cocaine-evoked [DA] to predict multiple addiction-like behaviors across various behavioral tests, both across all groups and within the IntA group, demonstrates that the magnitude of DA efflux in response to cocaine is an important factor in driving the expression of addiction-like behavior across all groups and not simply a function of self-administration history. One alternative interpretation of the microdialysis DA data is that the increased [DA] efflux in IntA rats, relative to LimA and $\operatorname{LgA}$ rats, was the result of increased locomotion following the cocaine infusion. However, we do not believe this is the case as the magnitude of [DA] efflux following the cocaine infusion was capable of predicting a number of addiction-like behaviors on a variety of behavioral procedures and changes in locomotion do not necessarily result in changes in [DA] levels in the nucleus accumbens (Paulson \& Robinson, 1994).

Active nose pokes during the no-drug period of IntA, $\alpha$, and cocaine-induced active nose pokes during the microdialysis test were used for the "IntA Addiction Score" analysis as they represent a diverse array of addiction-like behaviors, taken from different test procedures, that are relevant to the DSM V criteria for diagnosing substance use disorder in humans. Thus they represent a good index of general addiction-like behavior. There was similar variation in behavior between $\alpha$ and $P_{\text {Max }}$ within IntA rats and replacing $\alpha$ with $P_{\text {Max }}$ in the analysis yielded very similar results to those presented in Figure 7. We chose to show $\alpha$ for this analysis, as it is normalized with respect to $Q_{0}$ and thus slightly more informative in regard to differences in motivation than $P_{\text {Max }}$ alone (Bentzley et al., 2013, 2014).

These findings establish that IntA, a cocaine self-administration procedure that is especially effective in producing incentive-sensitization and addiction-like behavior, also sensitizes the dopaminergic response to cocaine. IntA also has been reported to be especially effective in producing a number of other neurobiological effects related to the development of addiction-like behavior, including dysregulation of mGluR2/3 receptor function (Allain et al., 2017), elevated BDNF levels (Gueye, Allain, \& Samaha, 2018), and increased activity in orexin/hypocretin neurons (James et al., 2018).

In contrast with the dopaminergic sensitization produced by IntA experience, there are a number of reports that $\operatorname{LgA}$ does the opposite-decreases DA function, relative to shorter access self-administration procedures. For example, following $\mathrm{LgA}$, or other high dose cocaine procedures, the ability of cocaine to inhibit DA uptake, or for electrical stimulation to evoke DA release from the accumbens core, is decreased in tissue slices, as is cocaine-evoked [DA] overflow measured with microdialysis in vivo (Calipari et al., 2013, 2014; Ferris et al., 2011; Siciliano, Fordahl, \& Jones, 2016). It may seem surprising, therefore, that in the present study a single, self-administered IV injection of cocaine increased [DA] to the same extent in rats with $\operatorname{LgA}$ or $\operatorname{LimA}$ experience-that is, there was no evidence for tolerance. It is not clear what accounts for the discrepancy-for example, ex vivo versus in vivo measures, experimenter-administered IP cocaine challenge vs. self-administered IV injection, measurement technique, or other methodological differences. However, the present results are consistent with one other study on the effects of $\operatorname{LgA}$ experience on [DA] measured with microdialysis in vivo. Ahmed et al. (2003) reported that, relative to a shorter access self-administration procedure, LgA did not decrease the [DA] response in the nucleus accumbens to either experimenter-administered IV injections of cocaine, or cocaine self-administration. Thus, it seems that $\operatorname{LgA}$ experience does not consistently decrease DA activity. It should also be noted that effects may vary considerably as a function of how long after the discontinuation of self-administration rats are tested (e.g., Ferrario et al., 2005; Siciliano et al., 2016). In addition, Willuhn et al. (2014) reported that the magnitude of a phasic DA response in the NAc following a nose poke that delivered cocaine progressively decreased with increasing $\mathrm{LgA}$ experience and was associated with the emergence of a DA signal in dorsal striatum, as measured with fast scan cyclic voltammetry. However, this phasic DA response peaked approximately $5 \mathrm{sec}$ after a nose poke, which is too soon to reflect the pharmacological effects of cocaine (Aragona et al., 2008; Stuber, Roitman, Phillips, Carelli, \& Wightman, 2005), and therefore, may not be relevant to the studies discussed above.

In addition to increasing extracellular [DA] levels, a single, self-administered cocaine infusion increased glutamate levels. Cocaine increases extracellular glutamate levels in the nucleus accumbens (Reid, Hsu, \& Berger, 1997; Smith, Mo, Guo, Kunko, \& Robinson, 1995). Interestingly, prolonged self-administration experience may decrease baseline glutamate levels, indicated by a trend toward elevated baseline levels in LimA rats compared to LgA and IntA rats, which 
did not differ. This is consistent with at least one previous study (Pierce, Bell, Duffy, \& Kalivas, 1996), although in this study cocaine-evoked glutatmate was greater in rats that had developed behavioral sensitization (Pierce et al., 1996) and we did not see any group differences in cocaine-evoked glutamate here. Nevertheless, this may be an interesting finding for future investigation.

Here, we also reported that a single, self-administered cocaine infusion increased 3-MT levels in all groups and 3-MT was increased to a greater extent in rats with IntA experience. DA is catalyzed to 3-MT by the enzyme cathechol-o-methyl transferase which is primarily localized extracellularly (Axelrod, 1966; Marsden, Broch, \& Guldberg, 1972). Therefore, manipulations that increase extracellular DA also increase 3-MT levels (Wood, Kim, \& Marien, 1987). That 3-MT levels were highest in rats with IntA experience supports the finidng that cocaine increased extracellular DA to the greatest extent in these rats, compared to rats with LimA or LgA experience.

One hour after the cocaine injection the cue that had been associated with cocaine was presented and we expected to see a conditioned DA response. But the cocaine cue had no effect in any group, on any neurochemical measure. It is not clear why this was the case, because the cue certainly had motivational properties, as indicated by the cue reinstatement test. However, if there were only a very brief (seconds) and relatively small response it may not have been detectable over the 3 min sampling period used here, and other techniques may be required to study the effects of IntA on such conditioned responses. This interpretation is supported by data showing that cues presented non-contingently elicit far less drug-seeking than cues presented contingently upon a drugseeking response (Kruzich, Congelton, \& See, 2001). Indeed, we did not see a behavioral response to the non-contingent cue presentation during the microdialysis test session.

\section{5 | CONCLUSIONS}

It has been suggested that addiction is characterized by a hypodopaminergic, anhedonic state, and compulsive motivation to seek and take cocaine derives from a desire to overcome this DA deficiency (Blum et al., 2015; Dackis \& Gold, 1985; Koob \& Le Moal, 1997, 2001; Volkow et al., 2016). Reports that $\mathrm{LgA}$ cocaine self-administration experience reduces DA function have been interpreted as support for this view, especially given that $\operatorname{LgA}$ was thought to best model changes in brain and behavior that lead to a transition from casual patterns of drug use to the escalated use that characterizes addiction. However, as reviewed above, the evidence that LgA produces a hypodopaminergic state is equivocal, as is evidence that it increases motivation for cocaine. In addition, studies using the more recently developed IntA self-administration procedure support a different theory. The IntA procedure was initially developed because it is thought to better model intermittent patterns of cocaine use in humans, especially during the transition to addiction (Allain et al., 2015; Zimmer et al., 2012). There is now considerable evidence that IntA produces incentive-sensitization, and is more effective than $\mathrm{LgA}$ in producing addiction-like behavior (Allain \& Samaha, 2018; Allain et al., 2017, 2018; James et al., 2018; Kawa \& Robinson, 2019; Kawa et al., 2016). Although the evidence is limited, and more work is required, the available evidence indicates that IntA experience also sensitizes DA function (Calipari et al., 2013, 2015), including the ability of cocaine to increase extracellular DA in vivo, as reported here.

In conclusion, studies utilizing the IntA procedure are more consistent with the view that the pathological motivation to seek and take cocaine in addiction is due, at least in part, to a hyper-responsive dopaminergic state, consistent with an incentive-sensitization view of addiction (Berridge \& Robinson, 2016; Robinson \& Berridge, 1993). Of course, a syndrome as complex as addiction is not going to be reducible to changes in a single neurotransmitter system, or even a single psychological process, and it remains to be seen what other neuropsychological functions are altered by IntA experience (e.g., Allain et al., 2017; Gueye et al., 2018; James et al., 2018). Nevertheless, the growing evidence concerning the importance of pharmacokinetic factors in promoting the development of addiction suggests these need to be given greater consideration in preclinical models of addiction (Allain et al., 2015; Kawa et al., 2019).

\section{ACKNOWLEDGEMENTS}

This research was supported by grants PO1 DA031656, RO1 DA044204 and T32 DA007281 from NIDA to TER and RO1 EB003320 to R.T.K.

\section{CONFLICT OF INTEREST}

The authors declare no conflict of interest.

\section{DATA ACCESSIBILITY}

Raw data generated by these experiments have been stored on a University of Michigan Server. Data and statistical analyses will be made available upon request.

\section{AUTHOR'S CONTRIBUTIONS}

ABK and TER designed the experiments with input from ACV and RTK, ABK collected the behavioral data and the microdialysis samples, ACV analyzed the microdialysis samples using an HPLC-MS. ABK, ACV, RTK, and TER 
analyzed the data, and $\mathrm{ABK}$ and TER wrote the manuscript with input from ACV and RTK.

\section{ORCID}

Alex B. Kawa (D) https://orcid.org/0000-0002-8870-0233

\section{REFERENCES}

Ahmed, S. H. (2012). The science of making drug-addicted animals. Neuroscience, 211, 107-125. https://doi.org/10.1016/j.neuroscien ce.2011.08.014

Ahmed, S. H. (2018). "A walk on the wild side" of addiction: The history and significance of animal models. In H. Pickard \& S. H. Ahmed (Eds.), Routledge handbook on philosophy and science of addiction (pp. 192 - 204). Routledge, NY: Routledge. https://doi. org/10.4324/9781315689197

Ahmed, S. H., \& Cador, M. (2006). Dissociation of psychomotor sensitization from compulsive cocaine consumption. Neuropsychopharmacology, 31, 563-571. https://doi.org/10.1038/ sj.npp. 1300834

Ahmed, S. H., \& Koob, G. F. (1998). Transition from moderate to excessive drug intake: Change in hedonic set point. Science, 282, 298300. https://doi.org/10.1126/science.282.5387.298

Ahmed, S. H., \& Koob, G. F. (1999). Long-lasting increase in the set point for cocaine self-administration after escalation in rats. Psychopharmacology (Berl), 146, 303-312. https://doi.org/10.1007/ s002130051121

Ahmed, S. H., Lin, D., Koob, G. F., \& Parsons, L. H. (2003). Escalation of cocaine self-administration does not depend on altered cocaine-induced nucleus accumbens dopamine levels. Journal of Neurochemistry, 86, 102-113.

Allain, F., Bouayad-Gervais, K., \& Samaha, A.-N. (2018). High and escalating levels of cocaine intake are dissociable from subsequent incentive motivation for the drug in rats. Psychopharmacology (Berl), 235, 317-328. https://doi.org/10.1007/s00213-017-4773-8

Allain, F., Minogianis, E.-A., Roberts, D. C. S., \& Samaha, A.-N. (2015). How fast and how often: The pharmacokinetics of drug use are decisive in addiction. Neuroscience and Biobehavioral Reviews, 56, 166-179. https://doi.org/10.1016/j.neubiorev.2015.06.012

Allain, F., Roberts, D. C. S., Lévesque, D., \& Samaha, A.-N. (2017). Intermittent intake of rapid cocaine injections promotes robust psychomotor sensitization, increased incentive motivation for the drug and mGlu2/3 receptor dysregulation. Neuropharmacology, 117, 227-237. https://doi.org/10.1016/j.neuropharm.2017.01.026

Allain, F., \& Samaha, A.-N. (2018). Revisiting long-access versus shortaccess cocaine self-administration in rats: Intermittent intake promotes addiction symptoms independent of session length. Addiction Biology in press. https://doi.org/10.1111/adb.12629.

Aragona, B. J., Cleaveland, N. A., Stuber, G. D., Day, J. J., Carelli, R. M., \& Wightman, R. M. (2008). Preferential enhancement of dopamine transmission within the nucleus accumbens shell by cocaine is attributable to a direct increase in phasic dopamine release events. Journal of Neuroscience, 28, 8821-8831. https://doi.org/10.1523/ JNEUROSCI.2225-08.2008

Axelrod, J. (1966). Methylation reactions in the formation and metabolism of catecholamines and other biogenic amines. Pharmacological Reviews, 18, 95-113.
Bentzley, B. S., Fender, K. M., \& Aston-Jones, G. (2013). The behavioral economics of drug self-administration: A review and new analytical approach for within-session procedures. Psychopharmacology (Berl), 226, 113-125. https://doi. org/10.1007/s00213-012-2899-2

Bentzley, B. S., Jhou, T. C., \& Aston-Jones, G. (2014). Economic demand predicts addiction-like behavior and therapeutic efficacy of oxytocin in the rat. Proceedings of the National Academy of Sciences, 111, 11822-11827. https://doi.org/10.1073/pnas.14063 24111

Berridge, K. C. (2004). Motivation concepts in behavioral neuroscience. Physiology \& Behavior, 81, 179-209. https://doi.org/10.1016/j. physbeh.2004.02.004

Berridge, K. C., \& Robinson, T. E. (2016). Liking, wanting, and the incentive-sensitization theory of addiction. American Psychologist, 71, 670-679. https://doi.org/10.1037/amp0000059

Blum, K., Thanos, P. K., Oscar-Berman, M., Febo, M., Baron, D., Badgaiyan, R. D., ... Gold, M. S. (2015). Dopamine in the brain: Hypothesizing surfeit or deficit links to reward and addiction. Journal of Reward Deficiency Syndrome, 1, 95-104.

Boyle, A. E., Gill, K., Smith, B. R., \& Amit, Z. (1991). Differential effects of an early housing manipulation on cocaine-induced activity and self-administration in laboratory rats. Pharmacology, Biochemistry and Behavior, 39, 269-274. https://doi. org/10.1016/0091-3057(91)90178-5

Bozarth, M. A., Murray, A., \& Wise, R. A. (1989). Influence of housing conditions on the acquisition of intravenous heroin and cocaine self-administration in rats. Pharmacology, Biochemistry and Behavior, 33, 903-907. https://doi. org/10.1016/0091-3057(89)90490-5

Calipari, E. S., Ferris, M. J., \& Jones, S. R. (2014). Extended access of cocaine self-administration results in tolerance to the dopamineelevating and locomotor-stimulating effects of cocaine. Journal of Neurochemistry, 128, 224-232. https://doi.org/10.1111/jnc.12452

Calipari, E. S., Ferris, M. J., Zimmer, B. A., Roberts, D. C. S., \& Jones, S. R. (2013). Temporal pattern of cocaine intake determines tolerance vs sensitization of cocaine effects at the dopamine transporter. Neuropsychopharmacology, 38, 2385-2392. https://doi. org/10.1038/npp.2013.136

Calipari, E. S., Siciliano, C. A., Zimmer, B. A., \& Jones, S. R. (2015). Brief intermittent cocaine self-administration and abstinence sensitizes cocaine effects on the dopamine transporter and increases drug seeking. Neuropsychopharmacology, 40, 728-735. https://doi. org/10.1038/npp.2014.238

Caprioli, D., Calu, D., \& Shaham, Y. (2014). Loss of phasic dopamine: A new addiction marker? Nature Neuroscience, 17, 644-646. https ://doi.org/10.1038/nn.3699

Carroll, M. E., France, C. P., \& Meisch, R. A. (1981). Intravenous selfadministration of etonitazene, cocaine and phencyclidine in rats during food deprivation and satiation. Journal of Pharmacology and Experimental Therapeutics, 217, 241-247.

Cohen, P., \& Sas, A. (1994). Cocaine use in Amsterdam in non deviant subcultures. Addiction Research and Theory, 2, 71-94. https://doi. org/10.3109/16066359409005547

Crombag, H. S., Badiani, A., Maren, S., \& Robinson, T. E. (2000). The role of contextual versus discrete drug-associated cues in promoting the induction of psychomotor sensitization to intravenous amphetamine. Behavioral Brain Research, 116, 1-22. https://doi. org/10.1016/S0166-4328(00)00243-6 
Dackis, C. A., \& Gold, M. S. (1985). New concepts in cocaine addiction: the dopamine depletion hypothesis. Neuroscience and Biobehavioral Reviews, 9, 469-477. https://doi.org/10.1016/01497634(85)90022-3

De Vry, J., Donselaar, I., \& Van Ree, J. M. (1989). Food deprivation and acquisition of intravenous cocaine self-administration in rats: Effect of naltrexone and haloperidol. Journal of Pharmacology and Experimental Therapeutics, 251, 735-740.

Deroche-Gamonet, V., Belin, D., \& Piazza, P. V. (2004). Evidence for addiction-like behavior in the rat. Science, 305, 1014-1017. https:// doi.org/10.1126/science.1099020

Edwards, S., \& Koob, G. F. (2013). Escalation of drug self-administration as a hallmark of persistent addiction liability. Behavioural Pharmacology, 24, 356-362. https://doi.org/10.1097/FBP.0b013 e3283644d 15

Ferrario, C. R., Gorny, G., Crombag, H. S., Li, Y., Kolb, B., \& Robinson, T. E. (2005). Neural and behavioral plasticity associated with the transition from controlled to escalated cocaine use. Biological Psychiatry, 58, 751-759. https://doi.org/10.1016/j.biops ych.2005.04.046

Ferris, M. J., Mateo, Y., Roberts, D. C. S., \& Jones, S. R. (2011). Cocaine-insensitive dopamine transporters with intact substrate transport produced by self-administration. Biological Psychiatry, 69, 201-207. https://doi.org/10.1016/j.biopsych.2010.06.026

Gerber, G. J., \& Wise, R. A. (1989). Pharmacological regulation of intravenous cocaine and heroin self-administration in rats: A variable dose paradigm. Pharmacology, Biochemistry and Behavior, 32, 527-531. https://doi.org/10.1016/0091-3057(89)90192-5

Gueye, A. B., Allain, F., \& Samaha, A.-N. (2018). Intermittent intake of rapid cocaine injections promotes the risk of relapse and increases mesocorticolimbic BDNF levels during abstinence. Neuropsychopharmacology, 44, 1027-1035. https://doi. org/10.1038/s41386-018-0249-8

Guillem, K., Ahmed, S. H., \& Peoples, L. L. (2014). Escalation of cocaine intake and incubation of cocaine seeking are correlated with dissociable neuronal processes in different accumbens subregions. Biological Psychiatry, 76, 31-39. https://doi.org/10.1016/j.biops ych.2013.08.032

Hershey, N. D., \& Kennedy, R. T. (2013). In vivo calibration of microdialysis using infusion of stable-isotope labeled neurotransmitters. ACS Chemical Neuroscience, 4, 729-736. https://doi.org/10.1021/ cn300199m

Hurd, Y. L., Weiss, F., Koob, G. F., And, N. E., \& Ungerstedt, U. (1989). Cocaine reinforcement and extracellular dopamine overflow in rat nucleus accumbens: An in vivo microdialysis study. Brain Research, 498, 199-203. https://doi.org/10.1016/0006-8993(89)90422-8

Hursh, S. R. (1991). Behavioral economics of drug self-administration and drug abuse policy. Journal of the Experimental Analysis of Behavior, 56, 377-393. https://doi.org/10.1901/jeab.1991.56-377

Hursh, S. R., \& Silberberg, A. (2008). Economic demand and essential value. Psychological Review, 115, 186-198. https://doi. org/10.1037/0033-295X.115.1.186

James, M. H., Stopper, C. M., Zimmer, B. A., Koll, N. E., Bowrey, H. E., \& Aston-Jones, G. (2018). Increased number and activity of a lateral subpopulation of hypothalamic orexin/hypocretin neurons underlies the expression of an addicted state in rats. Biological Psychiatry. https://doi.org/10.1016/j.biopsych.2018.07.022

Kawa, A. B., Allain, F., Robinson, T. E., \& Samaha, A.-N. (2019). The transition to cocaine addiction: The importance of pharmacokinetics for preclinical models. Psychopharmacology (Berl). https://doi. org/10.1007/s00213-019-5164-0

Kawa, A. B., Bentzley, B. S., \& Robinson, T. E. (2016). Less is more: Prolonged intermittent access cocaine self-administration produces incentive-sensitization and addiction-like behavior. Psychopharmacology (Berl), 233, 3587-3602. https://doi. org/10.1007/s00213-016-4393-8

Kawa, A. B., \& Robinson, T. E. (2019). Sex differences in incentivesensitization produced by intermittent access cocaine self-administration. Psychopharmacology (Berl), 236, 625-639. https://doi. org/10.1007/s00213-018-5091-5

Kippin, T. E., Fuchs, R. A., \& See, R. E. (2006). Contributions of prolonged contingent and noncontingent cocaine exposure to enhanced reinstatement of cocaine seeking in rats. Psychopharmacology (Berl), 187, 60-67. https://doi.org/10.1007/s00213-0060386-3

Koob, G. F., \& Le Moal, M. (1997). Drug abuse: Hedonic homeostatic dysregulation. Science, 278, 52-58. https://doi.org/10.1126/scien ce.278.5335.52

Koob, G. F., \& Le Moal, M. (2001). Drug addiction, dysregulation of reward, and allostasis. Neuropsychopharmacology, 24, 97-129. https ://doi.org/10.1016/S0893-133X(00)00195-0

Koob, G. F., \& Volkow, N. D. (2016). Neurobiology of addiction: A neurocircuitry analysis. The Lancet. Psychiatry, 3, 760-773. https:// doi.org/10.1016/S2215-0366(16)00104-8

Kruzich, P. J., Congelton, K. M., \& See, R. E. (2001). Conditioned reinstatement of drug-seeking behavior with a discrete compound stimulus classically conditioned with intravenous cocaine. Behavioral Neuroscience, 115, 1086-1092. https://doi. org/10.1037/0735-7044.115.5.1086

Liu, Y., Roberts, D. C. S., \& Morgan, D. (2005). Effects of extended-access self-administration and deprivation on breakpoints maintained by cocaine in rats. Psychopharmacology (Berl), 179, 644-651. https ://doi.org/10.1007/s00213-004-2089-y

Lu, L., Shepard, J. D., Scott Hall, F., \& Shaham, Y. (2003). Effect of environmental stressors on opiate and psychostimulant reinforcement, reinstatement and discrimination in rats: A review. Neuroscience and Biobehavioral Reviews, 27, 457-491. https://doi.org/10.1016/ S0149-7634(03)00073-3

Lynch, W. J., \& Carroll, M. E. (2001). Regulation of drug intake. Experimental and Clinical Psychopharmacology, 9, 131-143. https ://doi.org/10.1037/1064-1297.9.2.131

Mantsch, J. R., Baker, D. A., Francis, D. M., Katz, E. S., Hoks, M. A., $\&$ Serge, J. P. (2008). Stressor- and corticotropin releasing factor-induced reinstatement and active stress-related behavioral responses are augmented following long-access cocaine self-administration by rats. Psychopharmacology (Berl), 195, 591-603.

Mantsch, J. R., Yuferov, V., Mathieu-Kia, A. M., Ho, A., \& Kreek, M. J. (2004). Effects of extended access to high versus low cocaine doses on self-administration, cocaine-induced reinstatement and brain mRNA levels in rats. Psychopharmacology (Berl), 175, 26-36.

Marsden, C. A., Broch, O. J., \& Guldberg, H. C. (1972). Effect of nigral and raphé lesions on the catechol-O-methyl transferase and monoamine oxidase activities in the rat striatum. European Journal of Pharmacology, 19, 35-42. https://doi. org/10.1016/0014-2999(72)90074-X

Mateo, Y., Lack, C. M., Morgan, D., Roberts, D. C. S., \& Jones, S. R. (2005). Reduced dopamine terminal function and insensitivity to cocaine following cocaine binge self-administration and 
deprivation. Neuropsychopharmacology, 30, 1455-1463. https:// doi.org/10.1038/sj.npp.1300687

Nicola, S. M., \& Deadwyler, S. A. (2000). Firing rate of nucleus accumbens neurons is dopamine-dependent and reflects the timing of cocaine-seeking behavior in rats on a progressive ratio schedule of reinforcement. Journal of Neuroscience, 20, 5526-5537. https://doi. org/10.1523/JNEUROSCI.20-14-05526.2000

Oleson, E. B., Richardson, J. M., \& Roberts, D. C. S. (2011). A novel IV cocaine self-administration procedure in rats: Differential effects of dopamine, serotonin, and GABA drug pre-treatments on cocaine consumption and maximal price paid. Psychopharmacology (Berl), 214, 567-577. https://doi.org/10.1007/s00213-010-2058-6

Oleson, E. B., \& Roberts, D. C. S. (2009). Behavioral economic assessment of price and cocaine consumption following self-administration histories that produce escalation of either final ratios or intake. Neuropsychopharmacology, 34, 796-804. https://doi.org/10.1038/ npp. 2008.195

Paterson, N. E., \& Markou, A. (2003). Increased motivation for selfadministered cocaine after escalated cocaine intake. NeuroReport, 14, 2229-2232. https://doi.org/10.1097/00001756-20031 2020-00019

Paulson, P. E., \& Robinson, T. E. (1994). Relationship between circadian changes in spontaneous motor activity and dorsal versus ventral striatal dopamine neurotransmission assessed with on-line microdialysis. Behavioral Neuroscience, 108, 624-635. https://doi. org/10.1037/0735-7044.108.3.624

Paxinos, G., \& Watson, C. (2007). The rat brain in stereotaxic coordinates (6th ed.). San Diego, CA: Academic Press.

Phillips, G. D., Howes, S. R., Whitelaw, R. B., Wilkinson, L. S., Robbins, T. W., \& Everitt, B. J. (1994). Isolation rearing enhances the locomotor response to cocaine and a novel environment, but impairs the intravenous self-administration of cocaine. Psychopharmacology (Berl), 115, 407-418. https://doi.org/10.1007/BF02245084

Pierce, R., Bell, K., Duffy, P., \& Kalivas, P. (1996). Repeated cocaine augments excitatory amino acid transmission in the nucleus accumbens only in rats having developed behavioral sensitization. Journal of Neuroscience, 16, 1550-1560. https://doi.org/10.1523/ JNEUROSCI.16-04-01550.1996

Pitchers, K. K., Kane, L. F., Kim, Y., Robinson, T. E., \& Sarter, M. (2017). 'Hot' vs. 'cold' behavioural-cognitive styles: Motivationaldopaminergic vs. cognitive-cholinergic processing of a Pavlovian cocaine cue in sign- and goal-tracking rats. European Journal of Neuroscience, 46, 2768-2781. https://doi.org/10.1111/ejn.13741

Quadros, I. M. H., \& Miczek, K. A. (2009). Two modes of intense cocaine bingeing: Increased persistence after social defeat stress and increased rate of intake due to extended access conditions in rats. Psychopharmacology (Berl), 206, 109-120. https://doi.org/10.1007/ s00213-009-1584-6

Reid, M. S., Hsu, K., \& Berger, S. P.( 1997). Cocaine and amphetamine preferentially stimulate glutamate release in the limbic system: Studies on the involvement of dopamine. Synapse, 27, 95-105. https://doi.org/10.1002/(ISSN)1098-2396

Robinson, T. E., \& Berridge, K. C. (1993). The neural basis of drug craving: An incentive-sensitization theory of addiction. Brain Research Reviews, 18, 247-291. https://doi. org/10.1016/0165-0173(93)90013-P

Rowland, N. E. (2007). Food or fluid restriction in common laboratory animals: Balancing welfare considerations with scientific inquiry. Comparative Medicine, 57, 149-160.
Saunders, B. T., \& Robinson, T. E. (2010). A cocaine cue acts as an incentive stimulus in some but not others: Implications for addiction. Biological Psychiatry, 67, 730-736. https://doi.org/10.1016/j.biops ych.2009.11.015

Schenk, S., Lacelle, G., Gorman, K., \& Amit, Z. (1987). Cocaine selfadministration in rats influenced by environmental conditions: Implications for the etiology of drug abuse. Neuroscience Letters, 81, 227-231. https://doi.org/10.1016/0304-3940(87)91003-2

Sharpe, A. L., \& Samson, H. H. (2001). Effect of naloxone on appetitive and consummatory phases of ethanol self-administration. Alcoholism, Clinical and Experimental Research, 25, 1006-1011. https://doi.org/10.1111/j.1530-0277.2001.tb02309.x

Siciliano, C. A., Fordahl, S. C., \& Jones, S. R. (2016). Cocaine selfadministration produces long-lasting alterations in dopamine transporter responses to cocaine. Journal of Neuroscience, 36, 78077816. https://doi.org/10.1523/JNEUROSCI.4652-15.2016

Simon, S. L., Richardson, K., Dacey, J., Glynn, S., Domier, C. P., Rawson, R. A., \& Ling, W. (2002). A comparison of patterns of methamphetamine and cocaine use. Journal of Addictive Diseases, 21, 35-44.

Singer, B. F., Fadanelli, M., Kawa, A. B., \& Robinson, T. E. (2018). Are cocaine-seeking "habits" necessary for the development of addiction-like behavior in rats? Journal of Neuroscience, 38, 60-73.

Smith, J. A., Mo, Qiu., Guo, H., Kunko, P. M., \& Robinson, S. E. (1995). Cocaine increases extraneuronal levels of aspartate and glutamate in the nucleus accumbens. Brain Research, 683, 264-269. https://doi. org/10.1016/0006-8993(95)00383-2

Song, P., Mabrouk, O. S., Hershey, N. D., \& Kennedy, R. T. (2012). In vivo neurochemical monitoring using benzoyl chloride derivatization and liquid chromatography-mass spectrometry. Analytical Chemistry, 84, 412-419. https://doi.org/10.1021/ac202794q

Stuber, G. D., Roitman, M. F., Phillips, P. E. M., Carelli, R. M., \& Wightman, R. M. (2005). Rapid dopamine signaling in the nucleus accumbens during contingent and noncontingent cocaine administration. Neuropsychopharmacology, 30, 853-863. https://doi. org/10.1038/sj.npp.1300619

U.S. Department of Health and Human Services (2016). Facing addiction in America: The surgeon general's report on alcohol, drugs, and health. Washington DC: HHS.

Vanderschuren, L. J., \& Everitt, B. J. (2004). Drug seeking becomes compulsive after prolonged cocaine self-administration. Science, 305, 1017-1019. https://doi.org/10.1126/science.1098975

Volkow, N. D., Koob, G. F., \& McLellan, A. T. (2016). Neurobiologic advances from the brain disease model of addiction. New England Journal of Medicine, 374, 363-371. https://doi.org/10.1056/NEJMr a1511480

Wee, S., Mandyam, C. D., Lekic, D. M., \& Koob, G. F. (2008). Alpha 1-noradrenergic system role in increased motivation for cocaine intake in rats with prolonged access. European Neuropsychopharmacology, 18, 303-311. https://doi. org/10.1016/j.euroneuro.2007.08.003

Weeks, J. R. (1962). Experimental morphine addiction: Method for automatic intravenous injections in unrestrained rats. Science, 138, 143-144. https://doi.org/10.1126/science.138.3537.143

West, B. T., Welch, K. B., \& Galecki, A. T. (2007). Linear mixed models a practical guide using statistical software, statistics in medicine. Boca Raton, FL: Chapman \& Hall.

Willuhn, I., Burgeno, L. M., Groblewski, P. A., \& Phillips, P. E. M. (2014). Excessive cocaine use results from decreased phasic 
dopamine signaling in the striatum. Nature Neuroscience, 17, 704709. https://doi.org/10.1038/nn.3694

Wood, P. L., Kim, H. S., \& Marien, M. R. (1987). Intracerebral dialysis: Direct evidence for the utility of 3-MT measurements as an index of dopamine release. Life Sciences, 41, 1-5. https://doi. org/10.1016/0024-3205(87)90549-2

Xue, Y., Steketee, J. D., \& Sun, W. (2012). Inactivation of the central nucleus of the amygdala reduces the effect of punishment on cocaine self-administration in rats. European Journal of Neuroscience, 35, 775-783. https://doi.org/10.1111/j.1460-9568.2012.08000.x

Zimmer, B. A., Dobrin, C. V., \& Roberts, D. C. S. (2011). Braincocaine concentrations determine the dose self-administered by rats on a novel behaviorally dependent dosing schedule. Neuropsychopharmacology, 36, 2741-2749. https://doi. org/10.1038/npp.2011.165
Zimmer, B. A., Oleson, E. B., \& Roberts, D. C. (2012). The motivation to self-administer is increased after a history of spiking brain levels of cocaine. Neuropsychopharmacology, 37, 1901-1910. https://doi. org/10.1038/npp.2012.37

How to cite this article: Kawa AB, Valenta AC, Kennedy RT, Robinson TE. Incentive and dopamine sensitization produced by intermittent but not long access cocaine self-administration. Eur J Neurosci. 2019;50:2663-2682. https://doi.org/10.1111/ejn.14418 